ESAIM: PROCEEDINGS, April 2009, Vol. 26, p. 180-206

H. Ammari, Editor

\title{
MULTIPLE SCATTERING BY MULTIPLE SCATTERERS
}

\author{
P. A. MARTin ${ }^{1}$
}

\begin{abstract}
Acoustic scattering by random collections of identical circular cylinders is considered. Two classes of methods are used. The first is usually associated with the names of Foldy and Lax. Such methods require a "closure assumption", in addition to the governing equations. The second class is based on iterative approximations to integral equations of Lippmann-Schwinger type. Such methods do not use a closure approximation. Both methods are reviewed. In particular, when each cylinder is penetrable, with a sound-speed that is close to that in the exterior (the scattering is said to be "weak"), we show that both approaches lead to exactly the same formulas for the effective wavenumber, correct to second order in scattering strength and second order in filling fraction.
\end{abstract}

\section{INTRODUCTION}

Multiple scattering by multiple scatterers, arranged randomly, is a topic with an extensive literature and a wide range of applications. For references, see, for example, [11, 16, 21].

In this paper, we review some recent work on two-dimensional problems. A typical problem is the following. The region $x<0$ is filled with a homogeneous compressible fluid of density $\rho$ and sound-speed $c$. The region $x>0$ contains the same fluid and many scatterers. We suppose that the scatterers are identical circles. Then, a time-harmonic plane wave with wavenumber $k=\omega / c$ ( $\omega$ is the angular frequency) is incident on the scatterers: what is the reflected wave field? This field may be computed exactly for any given configuration of $N$ circles, but the cost increases with $N$. If the computation can be done, it may be repeated for other configurations, and then the average reflected field could be computed: this is the Monte Carlo approach.

Instead of adopting a purely computational approach, we shall use analytical methods. Two approaches will be discussed. The first leads to "Foldy-Lax" methods, and the second to iterative methods.

Foldy-Lax methods begin with a deterministic model for scattering by $N$ obstacles. Write such a model as

$$
u=u_{\text {in }}+\sum_{n=1}^{N} \mathcal{K}_{n} u_{n},
$$

where $u$ is the unknown wavefield, $u_{\text {in }}$ is the given incident field and $\mathcal{K}_{n}$ is an operator. The quantity $u_{n}$ can be viewed as the (unknown) contribution to $u$ coming from the scatterer centred at $\mathbf{r}_{n}$. Then, the ensemble average, $\langle u\rangle$, is calculated over all possible configurations of the scatterers. It follows from the right-hand side of (1) that we have to calculate $\left\langle\mathcal{K}_{n} u_{n}\right\rangle$, but this only exists if there is a scatterer at $\mathbf{r}_{n}$ : we are forced to introduce a conditional average of $u$. Thus, we cannot derive an equation for $\langle u\rangle$, merely a hierarchy of equations relating various different conditional averages of $u$. Breaking this hierarchy requires an additional "closure assumption". Such assumptions are difficult to justify, in general; for an example, see [25].

\footnotetext{
1 Department of Mathematical and Computer Sciences, Colorado School of Mines, Golden, Colorado 80401-1887, USA
}

(C) EDP Sciences, SMAI 2009 
For an alternative approach, suppose that we had an explicit formula for $u$, for any given configuration of the scatterers. Then, we could calculate $\langle u\rangle$ directly, in principle, without the use of closure assumptions. Of course, we do not have such an explicit formula for $u$, but we do have explicit approximations for $u$, and these could be used. This idea also has a long history; see, for example, [8] and [19, §7.4.2].

Both approaches will be discussed below. Both lead to formulas for the effective wavenumber $K$. This can then be used to replace the "random medium" occupying $x>0$ by a homogeneous "effective medium": the averaged field $\langle u\rangle$ is found to solve the Helmholtz equation, $\left(\nabla^{2}+K^{2}\right)\langle u\rangle=0$, in the fictitious effective medium.

\section{Foldy-Lax theories}

Foldy [6] began his analysis by considering isotropic point scatterers; this is an appropriate model for small sound-soft scatterers. He obtained the formula

$$
K^{2}=k^{2}-4 \mathrm{i} g n_{0},
$$

where $n_{0}$ is the number of circles per unit area and $g$ is the scattering coefficient for an individual scatterer. (In fact, Foldy considered scattering in three dimensions; the two-dimensional formula, (2), can be found as [22, eqn (3.20)] and [1, eqn (26)], for example.) The formula (2) assumes that the scatterers are independent, that $n_{0}$ is small, and that Foldy's closure assumption is valid. We are interested in calculating the correction to (2) (a term proportional to $n_{0}^{2}$ ), and this will require saying more about the distribution of the scatterers: we shall use pair correlations. Thus, our goal is a formula of the form

$$
K^{2}=k^{2}+\delta_{1} n_{0}+\delta_{2} n_{0}^{2}
$$

with computable expressions for $\delta_{1}$ and $\delta_{2}$. Also, we do not want to be limited to sound-soft scatterers.

In order to state formulas for $\delta_{1}$ and $\delta_{2}$, we introduce the far-field pattern $f$ for scattering by one circular cylinder. Thus, we have $u_{\text {in }}=\exp \left[\mathrm{i} k r \cos \left(\theta-\theta_{\text {in }}\right)\right]$ for the incident plane wave, where $(r, \theta)$ are plane polar coordinates $(x=r \cos \theta, y=r \sin \theta)$ and $\theta_{\text {in }}$ is the angle of incidence. The scattered waves satisfy

$$
u_{\mathrm{sc}} \sim \sqrt{2 /(\pi k r)} f\left(\theta-\theta_{\mathrm{in}}\right) \exp (\mathrm{i} k r-\mathrm{i} \pi / 4) \quad \text { as } r \rightarrow \infty .
$$

Foldy-type theories use his closure assumption, they are essentially linear in $n_{0}$, they assume that the scatterers are independent, and they predict that

$$
K^{2}=k^{2}-4 \operatorname{iin} n_{0}(0)
$$

(identifying $g$ in (2)). Also, when $u_{\text {in }}=\mathrm{e}^{\mathrm{i} k x}$,

$$
\langle u\rangle=A \mathrm{e}^{\mathrm{i} K x},
$$

where Foldy theory predicts that the "amplitude", $A$, is given by

$$
A=1+\mathrm{i} n_{0} k^{-2} f(0) .
$$

Observe that if we write $A=|A| \mathrm{e}^{\mathrm{i} \alpha}$ and $K=K_{\mathrm{r}}+\mathrm{i} K_{\mathrm{i}}$, then $\operatorname{Re}\left\{\langle u\rangle \mathrm{e}^{-\mathrm{i} \omega t}\right\}=|A| \mathrm{e}^{-K_{\mathrm{i}} x} \cos \left(K_{\mathrm{r}} x-\omega t+\alpha\right)$, showing that the quantities $|A|$ and $K_{\mathrm{i}}=\operatorname{Im} K$ are of interest.

Returning to (3), we note that there is some controversy over the proper value for $\delta_{2}$. Twersky [22] has given the following formula:

$$
K^{2}=k^{2}-4 \operatorname{in} n_{0} f(0)+\left(2 n_{0} / k\right)^{2} \sec ^{2} \theta_{\text {in }}\left\{\left[f\left(\pi-2 \theta_{\text {in }}\right)\right]^{2}-[f(0)]^{2}\right\} .
$$

This formula involves $\theta_{\text {in }}$, so that it gives a different effective wavenumber for different incident fields. 
The three-dimensional version of (8) is older. For a random collection of identical spheres, it is

$$
K^{2}=k^{2}-4 \pi \mathrm{i}\left(\hat{n}_{0} / k\right) f(0)+\delta_{2} \hat{n}_{0}^{2}
$$

with $[22]$

$$
\delta_{2}=\left(4 \pi^{2} / k^{4}\right) \sec ^{2} \theta_{\text {in }}\left\{\left[f\left(\pi-2 \theta_{\text {in }}\right)\right]^{2}-[f(0)]^{2}\right\},
$$

where $f$ is now defined by $u_{\mathrm{sc}} \sim(\mathrm{i} k r)^{-1} \mathrm{e}^{\mathrm{i} k r} f(\vartheta), r$ and $\vartheta$ are spherical polar coordinates, and $\hat{n}_{0}$ is the number of spheres per unit volume. The same formula but with $\theta_{\text {in }}=0$ (normal incidence) was given by Waterman and Truell [24]. However, it was shown by Lloyd and Berry in 1967 [14] that (9) is incorrect; they obtained

$$
\delta_{2}=\frac{4 \pi^{2}}{k^{4}}\left\{-[f(\pi)]^{2}+[f(0)]^{2}+\int_{0}^{\pi} \frac{1}{\sin (\vartheta / 2)} \frac{\mathrm{d}}{\mathrm{d} \vartheta}[f(\vartheta)]^{2} \mathrm{~d} \vartheta\right\}
$$

(with no dependence on $\theta_{\text {in }}$ ). Lloyd and Berry [14] used methods (and language) coming from nuclear physics. Thus, in their approach, which they "call the 'resummation method', a point source of waves is considered to be situated in an infinite medium. The scattering series is then written out completely, giving what Lax has called the 'expanded' representation. In this expanded representation the ensemble average may be taken exactly [but then] the coherent wave does not exist; the series must be resummed in order to obtain any result at all". Linton and Martin [11] have shown that a proper analysis of the semi-infinite two-dimensional model problem (with arbitrary angle of incidence) leads to a formula that is reminiscent of the (three-dimensional) Lloyd-Berry formula; specifically, instead of (8), they obtained

$$
K^{2}=k^{2}-4 \mathrm{i} n_{0} f(0)+\frac{8 n_{0}^{2}}{\pi k^{2}} \int_{0}^{\pi} \cot (\theta / 2) \frac{\mathrm{d}}{\mathrm{d} \theta}[f(\theta)]^{2} \mathrm{~d} \theta .
$$

Their analysis does not involve "resumming" series or divergent integrals. It uses a classical approach, in the spirit of the papers by Fikioris and Waterman [5] and by Bose and Mal [2], and it uses the Lax quasicrystalline approximation (QCA) as closure assumption. Linton and Martin [12] have also given a similar analysis in three dimensions: it leads to a new proof of the Lloyd-Berry formula, (10).

Calculations at second order in $n_{0}$ are more difficult because one has to introduce conditional probabilities, intended to prevent scatterers overlapping during the averaging process. We use a simple pair-correlation function, giving what is known as a "hole correction". This involves a new parameter, $b$, with $b \geq 2 a$. If conditional probabilities are not used, then the scatterers are essentially independent and so one should not expect to do better than Foldy's theory, which is linear in $n_{0}$. Note that the formula (11) does not involve $b$; its fairly complicated form comes by taking the limit $b \rightarrow 0$ in another formula for $\delta_{2}$, one that holds for finite $b$; see (65).

The analysis of Linton and Martin [11] will be summarised in Section 3. For simplicity, we assume normal incidence in the calculations; see the original paper for non-normal incidence.

\section{Iterative theories}

The theories of Foldy-Lax type, described above, are relatively insensitive to the composition of the cylinders: they can be penetrable or impenetrable. If penetrable, the wavenumber $\left(k_{0}\right)$ and density $\left(\rho_{0}\right)$ inside can differ from the wavenumber $(k)$ and density $(\rho)$ outside. In order to obtain a plausible iterative theory, we assume that the scattering is weak, which we define as $\rho=\rho_{0}$ and $k \simeq k_{0}$. Specifically, we assume that the strength $m_{0}=1-\left(k_{0} / k\right)^{2}$ is small. This assumption is convenient for the derivation of good approximations to the deterministic problem. We shall work to second order in both $m_{0}$ and $n_{0}$.

As $\rho=\rho_{0}$ (the fluid density is constant everywhere), the deterministic scattering problem can be solved using the Lippmann-Schwinger integral equation; see Section 5. (This equation can also be used when $k_{0}$ is a function of position; if $\rho \neq \rho_{0}$, a different integral equation must be used [15].) Under certain circumstances, the Lippmann-Schwinger equation can be solved by iteration; we examine the first-order (Born) approximation (linear in the strength $m_{0}$ ) and the second-order approximation (quadratic in $m_{0}$ ). For one circular scatterer, 
this is done in Section 6. We confirm that the second-order iterative approximation for the wavefield (both inside and outside the scatterer) agrees with the second-order approximation of the exact solution.

Scattering by random arrangements of scatterers is considered in Section 7. We write down the iterative approximation for scattering by $N$ circles. Then, we calculate the ensemble average; we begin with first order in both $n_{0}$ and $m_{0}$, and end with second order in both. No closure assumptions are used. At first order in $n_{0}$, we find precise agreement with the Foldy estimate for $K,(5)$, correct to second order in $m_{0}$. For the amplitude, $A$, we find agreement at first order in $m_{0}$ with the Foldy estimate (given by $(7)$ ), but we find a discrepancy at second order; see (146).

At second order in $n_{0}$, we use the "hole correction". We have the finite- $b$ formula for $\delta_{2}$ in (3) found by Linton and Martin [11], making use of the Lax QCA for their closure assumption; see Section 4.3. We compare this formula (approximated to second order in $m_{0}$ ) with the iterative approach: the agreement turns out to be perfect. One could view this agreement as supporting either of the two approaches described above, depending on one's point of view. We also obtain a new estimate for $A$, and we verify agreement with known results for "point scatterers" [18] in the appropriate limit; see Section 7.3.

\section{Outline}

In outline, the paper is as follows. Some elementary probability theory is recalled in Section 1 . The paircorrelation function is introduced; this leads to the "hole correction" - individual cylinders must not be allowed to overlap during the averaging process. In Section 2, we derive the integral equations of Foldy (isotropic scatterers, no hole correction) and of Lax (isotropic scatterers, hole correction included). Foldy's integral equation can be solved exactly whereas we have been unable to solve Lax's integral equation. Nevertheless, we have developed a rigorous method for extracting an expression for $K$ (but not $A$ ) from these integral equations without actually solving the integral equations themselves. Then, we use the same method in Section 3 but without the restriction to isotropic scatterers. We start by following Bose and Mal [2], and use an exact (deterministic) theory for scattering by $N$ circles followed by ensemble averaging. We give a clear derivation of a certain homogeneous infinite linear system of algebraic equations, obtained previously by Bose and Mal [2]; the existence of a non-trivial solution determines $K$. We solve the system for small $n_{0}$, and obtain two formulas for $K$, including (11). We have also shown that (8) is obtained if the hole correction is not made correctly.

Section 4 is a transitional section. We take the exact solution for scattering by one circular penetrable cylinder, and then approximate assuming that the scattering is weak. This leads to weak-scattering versions of the Foldy-Lax theories (including the Linton-Martin theory) described above.

The Lippmann-Schwinger integral equation and its iterative approximations are given in Section 5 . Results for one cylinder are obtained in Section 6. Section 7 is dedicated to random collections of cylinders. Detailed comparisons between the two approaches (Foldy-Lax and iterative) are made. Maurel [18] has made similar comparisons, but with uncorrelated "point scatterers", meaning that each scatterer is represented by a Dirac delta function; we are interested here in scatterers of finite size (and we do not make small- $k a$ approximations). Comparisons with Maurel's results are made in Section 7.3. The paper ends with a summary in Section 8.

\section{Some PROBABILITy THEORY}

We give a brief summary of the probability theory needed. For more information, see $[1,6,9]$.

Suppose we have $N$ scatterers located at the points $\mathbf{r}_{1}, \mathbf{r}_{2}, \ldots, \mathbf{r}_{N}$ inside a region $B_{N}$; denote the configuration of points by $\Lambda_{N}=\left\{\mathbf{r}_{1}, \mathbf{r}_{2}, \ldots, \mathbf{r}_{N}\right\}$. Then, the ensemble average of any quantity $F\left(\mathbf{r} \mid \Lambda_{N}\right)$ is defined by

$$
\langle F(\mathbf{r})\rangle=\int \cdots \int p\left(\mathbf{r}_{1}, \mathbf{r}_{2}, \ldots, \mathbf{r}_{N}\right) F\left(\mathbf{r} \mid \Lambda_{N}\right) \mathrm{d} V_{1} \cdots \mathrm{d} V_{N}
$$

where the the integration is over $N$ copies of $B_{N}$. Here, $p\left(\mathbf{r}_{1}, \ldots, \mathbf{r}_{N}\right) \mathrm{d} V_{1} \mathrm{~d} V_{2} \cdots \mathrm{d} V_{N}$ is the probability of finding the scatterers in a configuration in which the first scatterer is in the element $\mathrm{d} V_{1}$ about $\mathbf{r}_{1}$, the second scatterer is in the element $\mathrm{d} V_{2}$ about $\mathbf{r}_{2}$, and so on, up to $\mathbf{r}_{N}$. The joint probability distribution $p\left(\mathbf{r}_{1}, \ldots, \mathbf{r}_{N}\right)$ is 
normalised so that $\langle 1\rangle=1$. Similarly, the average of $F\left(\mathbf{r} \mid \Lambda_{N}\right)$ over all configurations for which the first scatterer is fixed at $\mathbf{r}_{1}$ is given by

$$
\langle F(\mathbf{r})\rangle_{1}=\int \cdots \int p\left(\mathbf{r}_{2}, \ldots, \mathbf{r}_{N} \mid \mathbf{r}_{1}\right) F\left(\mathbf{r} \mid \Lambda_{N}\right) \mathrm{d} V_{2} \cdots \mathrm{d} V_{N},
$$

where $p\left(\mathbf{r}_{1}, \mathbf{r}_{2}, \ldots, \mathbf{r}_{N}\right)=p\left(\mathbf{r}_{1}\right) p\left(\mathbf{r}_{2}, \ldots, \mathbf{r}_{N} \mid \mathbf{r}_{1}\right)$ defines the conditional probability $p\left(\mathbf{r}_{2}, \ldots, \mathbf{r}_{N} \mid \mathbf{r}_{1}\right)$. If two scatterers are fixed, say the first and the second, we can define

$$
\langle F(\mathbf{r})\rangle_{12}=\int \cdots \int p\left(\mathbf{r}_{3}, \ldots, \mathbf{r}_{N} \mid \mathbf{r}_{1}, \mathbf{r}_{2}\right) F\left(\mathbf{r} \mid \Lambda_{N}\right) \mathrm{d} V_{3} \cdots \mathrm{d} V_{N},
$$

where $p\left(\mathbf{r}_{2}, \ldots, \mathbf{r}_{N} \mid \mathbf{r}_{1}\right)=p\left(\mathbf{r}_{2} \mid \mathbf{r}_{1}\right) p\left(\mathbf{r}_{3}, \ldots, \mathbf{r}_{N} \mid \mathbf{r}_{1}, \mathbf{r}_{2}\right)$.

Now, as each of the $N$ scatterers is equally likely to occupy $\mathrm{d} V_{1}$, the density of scatterers at $\mathbf{r}_{1}$ is $N p\left(\mathbf{r}_{1}\right)=n_{0}$, the (constant) number of scatterers per unit volume. Thus

$$
p(\mathbf{r})=n_{0} / N=\left|B_{N}\right|^{-1},
$$

where $\left|B_{N}\right|$ is the area of $B_{N}$. Also, as $p\left(\mathbf{r}_{1}, \mathbf{r}_{2}\right)=p\left(\mathbf{r}_{1}\right) p\left(\mathbf{r}_{2} \mid \mathbf{r}_{1}\right)$, we obtain

$$
\iint p\left(\mathbf{r}_{2} \mid \mathbf{r}_{1}\right) \mathrm{d} V_{1} \mathrm{~d} V_{2}=\frac{N}{n_{0}}=\left|B_{N}\right| .
$$

We specify $p\left(\mathbf{r}_{2} \mid \mathbf{r}_{1}\right)$ consistent with (16). Also, we want to ensure that scatterers do not overlap. For circular cylinders of radius $a$, a simple choice is $p\left(\mathbf{r}_{2} \mid \mathbf{r}_{1}\right)=p_{0} H\left(R_{12}-b\right)$ with $b \geq 2 a$, where $H(x)$ is the Heaviside unit function, $R_{12}=\left|\mathbf{r}_{1}-\mathbf{r}_{2}\right|$ and $p_{0}$ is a constant determined by (16). Thus, $p_{0}=\left\{\left|B_{N}\right|-\pi b^{2}\right\}^{-1} \simeq n_{0} / N$, assuming that $b^{2} n_{0} / N \ll 1$. Hence, the simplest sensible choice for the pair-correlation function is

$$
p\left(\mathbf{r}_{2} \mid \mathbf{r}_{1}\right)= \begin{cases}0, & R_{12}<b \\ n_{0} / N, & R_{12}>b .\end{cases}
$$

This simple choice will be used for throughout our analysis. More generally, we could use

$$
p\left(\mathbf{r}_{2} \mid \mathbf{r}_{1}\right)=\left(n_{0} / N\right)\left[1+\chi\left(R_{12} ; n_{0}\right)\right] H\left(R_{12}-b\right),
$$

where the function $\chi$ is to be chosen, subject to some constraints.

\section{FOLDY-LAX THEORY: ISOTROPIC SCATTERERS}

Foldy's theory begins with a simplified deterministic model for scattering by $N$ identical scatterers, each of which is supposed to scatter isotropically. Thus, the total field is assumed to be given by the incident field plus a point source at each scattering centre, $\mathbf{r}_{j}$ :

$$
u\left(\mathbf{r} \mid \Lambda_{N}\right)=u_{\text {in }}(\mathbf{r})+g \sum_{j=1}^{N} u_{\mathrm{ex}}\left(\mathbf{r}_{j} ; \mathbf{r}_{j} \mid \Lambda_{N}\right) H_{0}\left(k\left|\mathbf{r}-\mathbf{r}_{j}\right|\right) .
$$

Here, $H_{n}(w) \equiv H_{n}^{(1)}(w)$ is a Hankel function, $g$ is the (assumed known) scattering coefficient, and the exciting field $u_{\text {ex }}$ is given by

$$
u_{\mathrm{ex}}\left(\mathbf{r} ; \mathbf{r}_{n} \mid \Lambda_{N}\right)=u_{\mathrm{in}}(\mathbf{r})+g \sum_{\substack{j=1 \\ j \neq n}}^{N} u_{\mathrm{ex}}\left(\mathbf{r}_{j} ; \mathbf{r}_{j} \mid \Lambda_{N}\right) H_{0}\left(k\left|\mathbf{r}-\mathbf{r}_{j}\right|\right) .
$$


The second term in (20) is the field near the cylinder at $\mathbf{r}_{n}$ due to scattering by all the other cylinders. The $N$ numbers $u_{\mathrm{ex}}\left(\mathbf{r}_{j} ; \mathbf{r}_{j} \mid \Lambda_{N}\right)(j=1,2, \ldots, N)$ required in (19) are to be determined by solving the linear system obtained by evaluating (20) at $\mathbf{r}=\mathbf{r}_{n}$.

Let us try to compute the ensemble average of $u$, using (19) and (12). The result is

$$
\langle u(\mathbf{r})\rangle=u_{\text {in }}(\mathbf{r})+g n_{0} \int_{B_{N}}\left\langle u_{\text {ex }}\left(\mathbf{r}_{1}\right)\right\rangle_{1} H_{0}\left(k\left|\mathbf{r}-\mathbf{r}_{1}\right|\right) \mathrm{d} V_{1},
$$

where we have used (13) and (15), and the indistinguishability of the scatterers. For $\left\langle u_{\mathrm{ex}}\left(\mathbf{r}_{1}\right)\right\rangle_{1}$ (which is given explicitly by (13) in which $u_{\mathrm{ex}}\left(\mathbf{r}_{1} ; \mathbf{r}_{1} \mid \Lambda_{N}\right)$ is substituted for $\left.F\left(\mathbf{r} \mid \Lambda_{N}\right)\right)$, we obtain

$$
\left\langle u_{\text {ex }}(\mathbf{r})\right\rangle_{1}=u_{\text {in }}(\mathbf{r})+g(N-1) \int_{B_{N}} p\left(\mathbf{r}_{2} \mid \mathbf{r}_{1}\right)\left\langle u_{\text {ex }}\left(\mathbf{r}_{2}\right)\right\rangle_{12} H_{0}\left(k\left|\mathbf{r}-\mathbf{r}_{2}\right|\right) \mathrm{d} V_{2},
$$

where we have used (14) and (20). Equations (21) and (22) are the first two in a hierarchy, involving more and more complicated information on the statistics of the scatterer distribution. In practice, the hierarchy is broken using an additional "closure assumption". At the lowest level, we have Foldy's closure assumption,

$$
\left\langle u_{\mathrm{ex}}(\mathbf{r})\right\rangle_{1} \simeq\langle u(\mathbf{r})\rangle
$$

at least in the neighbourhood of $\mathbf{r}_{1}$. When this is used in (21), we obtain

$$
\langle u(\mathbf{r})\rangle=u_{\text {in }}(\mathbf{r})+g n_{0} \int_{B_{N}}\left\langle u\left(\mathbf{r}_{1}\right)\right\rangle H_{0}\left(k\left|\mathbf{r}-\mathbf{r}_{1}\right|\right) \mathrm{d} V_{1}, \quad \mathbf{r} \in B_{N} .
$$

We call this Foldy's integral equation for $\langle u\rangle$. The integral on the right-hand side is an acoustic volume potential. Hence, an application of $\left(\nabla^{2}+k^{2}\right)$ to $(24)$ eliminates the incident field and shows that $\left(\nabla^{2}+K^{2}\right)\langle u\rangle=0$ in $B_{N}$, where $K^{2}$ is given by Foldy's formula, (2).

At the next level, we have the Lax [10] quasi-crystalline assumption (QCA),

$$
\left\langle u_{\mathrm{ex}}(\mathbf{r})\right\rangle_{12} \simeq\left\langle u_{\mathrm{ex}}(\mathbf{r})\right\rangle_{2} .
$$

When this is used in (22) evaluated at $\mathbf{r}=\mathbf{r}_{1}$, we obtain

$$
v(\mathbf{r})=u_{\text {in }}(\mathbf{r})+g(N-1) \int_{B_{N}} p\left(\mathbf{r}_{1} \mid \mathbf{r}\right) v\left(\mathbf{r}_{1}\right) H_{0}\left(k\left|\mathbf{r}-\mathbf{r}_{1}\right|\right) \mathrm{d} V_{1}, \quad \mathbf{r} \in B_{N},
$$

where $v(\mathbf{r})=\left\langle u_{\mathrm{ex}}(\mathbf{r})\right\rangle_{1}$. We call this Lax's integral equation.

In what follows, we let $N \rightarrow \infty$ so that $B_{N} \rightarrow B_{\infty}$, a semi-infinite region, $x>0$.

\subsection{Foldy's integral equation: exact treatment}

Consider a plane wave at normal incidence, so that $u_{\text {in }}=\mathrm{e}^{\mathrm{i} k x}$. For a semi-infinite domain $B_{\infty}$, Foldy's integral equation, (24), becomes

$$
\langle u(x, y)\rangle=\mathrm{e}^{\mathrm{i} k x}+g n_{0} \int_{0}^{\infty} \int_{-\infty}^{\infty}\left\langle u\left(x_{1}, y+Y\right)\right\rangle H_{0}\left(k \rho_{1}\right) \mathrm{d} Y \mathrm{~d} x_{1}, \quad \begin{gathered}
x>0, \\
-\infty<y<\infty
\end{gathered}
$$

where $\rho_{1}=\sqrt{\left(x-x_{1}\right)^{2}+Y^{2}}$. This equation can be solved exactly. Thus, writing

$$
\langle u(x, y)\rangle=U(x), \quad x>0, \quad-\infty<y<\infty
$$


we obtain

$$
U(x)=\mathrm{e}^{\mathrm{i} k x}+g n_{0} \int_{0}^{\infty} \int_{-\infty}^{\infty} U\left(x_{1}\right) H_{0}\left(k \rho_{1}\right) \mathrm{d} Y \mathrm{~d} x_{1}, \quad \begin{gathered}
x>0, \\
-\infty<y<\infty .
\end{gathered}
$$

Now,

$$
\int_{-\infty}^{\infty} H_{0}\left(k \rho_{1}\right) \mathrm{d} Y \frac{2}{k} \mathrm{e}^{\mathrm{i} k\left|x-x_{1}\right|},
$$

whence (28) simplifies to

$$
U(x)=\mathrm{e}^{\mathrm{i} k x}+\frac{2 g n_{0}}{k} \int_{0}^{\infty} U\left(x_{1}\right) \mathrm{e}^{\mathrm{i} k\left|x-x_{1}\right|} \mathrm{d} x_{1}, \quad x>0 .
$$

Now, put $U(x)=U_{0} \mathrm{e}^{\mathrm{i} K x}$, so that (27) gives

$$
\langle u(x, y)\rangle=U_{0} \mathrm{e}^{\mathrm{i} K x}, \quad x>0, \quad-\infty<y<\infty,
$$

and (30) gives

$$
U_{0} \mathrm{e}^{\mathrm{i} K x}-\mathrm{e}^{\mathrm{i} k x}=\frac{2 g n_{0} U_{0}}{\mathrm{i} k}\left(\frac{2 k \mathrm{e}^{\mathrm{i} K x}}{K^{2}-k^{2}}-\frac{\mathrm{e}^{\mathrm{i} k x}}{K-k}\right),
$$

where we have assumed that $\operatorname{Im} K>0$. If we compare the coefficients of $\mathrm{e}^{\mathrm{i} K x}$, we see that $U_{0}$ cancels, leaving Foldy's formula, (2). Then, the coefficients of $\mathrm{e}^{\mathrm{i} k x}$ give $U_{0}=2 k /(K+k)$. A similar method can be used to find $\langle u\rangle$ when $B_{\infty}$ is a slab of finite thickness, $0<x<L$, say; see [1].

\subsection{Foldy's integral equation: approximate treatment}

We have seen that Foldy's integral equation can be solved exactly, and that the solution process has two parts: first find $K$ and then find $U_{0}$. In fact, $K$ can be found without finding the complete solution; the reason for pursuing this is that we cannot usually find exact solutions. Thus, consider (30), and suppose that

$$
U(x)=U_{0} \mathrm{e}^{\mathrm{i} K x} \quad \text { for } x>\ell,
$$

where $U_{0}, K$ and $\ell$ are unknown. To proceed, we need say nothing about the solution $U$ in the "boundary layer" $0<x<\ell$. Now, evaluate the integral equation for $x>\ell$; we find that

$$
U_{0} \mathrm{e}^{\mathrm{i} K x}-\mathrm{e}^{\mathrm{i} k x} \frac{2 g n_{0}}{k} \mathrm{e}^{\mathrm{i} k x} \int_{0}^{\ell} U(t) \mathrm{e}^{-\mathrm{i} k t} \mathrm{~d} t+\frac{2 g n_{0}}{k} \int_{\ell}^{\infty} U(t) \mathrm{e}^{\mathrm{i} k|x-t|} \mathrm{d} t=\mathcal{A} \mathrm{e}^{\mathrm{i} K x}+\mathcal{B} \mathrm{e}^{\mathrm{i} k x}
$$

for $x>\ell$, where $\mathcal{A}=-4 \mathrm{ign} n_{0} U_{0} /\left(K^{2}-k^{2}\right)$ and

$$
\mathcal{B}=\frac{2 g n_{0}}{k} \int_{0}^{\ell} U(t) \mathrm{e}^{-\mathrm{i} k t} \mathrm{~d} t+\frac{2 \mathrm{i} g n_{0} U_{0}}{k(K-k)} \mathrm{e}^{\mathrm{i}(K-k) \ell} .
$$

Then, setting $U_{0}=\mathcal{A}$ gives (2) again, without knowing the solution $U$ everywhere. This basic method will be used again below.

\subsection{Lax's integral equation}

Using the approximation $p\left(\mathbf{r}_{1} \mid \mathbf{r}\right)=\left(n_{0} / N\right) H\left(R_{1}-b\right)$ in Lax's integral equation, (26) gives

$$
v(\mathbf{r})=u_{\text {in }}(\mathbf{r})+g n_{0} \frac{N-1}{N} \int_{B_{N}^{b}(\mathbf{r})} v\left(\mathbf{r}_{1}\right) H_{0}\left(k R_{1}\right) \mathrm{d} \mathbf{r}_{1}, \quad \mathbf{r} \in B_{N},
$$

where $B_{N}^{b}(\mathbf{r})=\left\{\mathbf{r}_{1} \in B_{N}: R_{1}=\left|\mathbf{r}-\mathbf{r}_{1}\right|>b\right\}$, which is $B_{N}$ with a (possibly incomplete) disc excluded. 
Let $N \rightarrow \infty$ and take an incident plane wave giving

$$
v(x, y)=\mathrm{e}^{\mathrm{i} k x}+g n_{0} \int_{x_{1}>0, \rho_{1}>b} v\left(x_{1}, y+Y\right) H_{0}\left(k \rho_{1}\right) \mathrm{d} Y \mathrm{~d} x_{1}, \quad x>0,-\infty<y<\infty .
$$

As in Section 2.1, we write $v(x, y)=V(x)$ for $x>0$ and all $y$, giving

$$
V(x)=\mathrm{e}^{\mathrm{i} k x}+g n_{0} \int_{x_{1}>0, \rho_{1}>b} V\left(x_{1}\right) H_{0}\left(k \rho_{1}\right) \mathrm{d} Y \mathrm{~d} x_{1}, \quad x>0 .
$$

Then, using (29), we see that $V$ solves

$$
V(x)=\mathrm{e}^{\mathrm{i} k x}+g n_{0} \int_{0}^{\infty} V\left(x_{1}\right) L\left(x-x_{1}\right) \mathrm{d} x_{1}, \quad x>0,
$$

where the kernel, $L\left(x-x_{1}\right)$, is given by

$$
L(X)=\frac{2}{k} \mathrm{e}^{\mathrm{i} k|X|}-2 \int_{0}^{c(X)} H_{0}\left(k \sqrt{X^{2}+Y^{2}}\right) \mathrm{d} Y \quad \text { with } \quad c(X)=\sqrt{b^{2}-X^{2}} H(b-|X|) .
$$

We have been unable to solve (34) exactly (even though it is an integral equation of Wiener-Hopf type). However, the approximate method described in Section 2.2 can be used. Thus, let us suppose that

$$
V(x)=V_{0} \mathrm{e}^{\mathrm{i} K x} \quad \text { for } x>\ell
$$

where $V_{0}, K$ and $\ell$ are unknown. Then, consider (34) for $x>\ell+b$, so that the interval $\left|x-x_{1}\right|<b$ is entirely within the range $x_{1}>\ell$. Making use of (35), (34) gives

$$
\begin{aligned}
& \frac{V_{0} \mathrm{e}^{\mathrm{i} K x}-\mathrm{e}^{\mathrm{i} k x}}{g n_{0}} \frac{2}{k} \mathrm{e}^{\mathrm{i} k x} \int_{0}^{\ell} V(t) \mathrm{e}^{-\mathrm{i} k t} \mathrm{~d} t+\frac{2}{k} \int_{\ell}^{\infty} V(t) \mathrm{e}^{\mathrm{i} k|x-t|} \mathrm{d} t \\
& -2 \int_{x-b}^{x+b} V(t) \int_{0}^{c(x-t)} H_{0}\left(k \sqrt{(x-t)^{2}+Y^{2}}\right) \mathrm{d} Y \mathrm{~d} t \quad \text { for } x>\ell+b .
\end{aligned}
$$

Equation (36) can be used in the second and third integrals. The second integral is elementary; its value is

$$
\frac{2 \mathrm{i} V_{0}}{k(K-k)} \mathrm{e}^{\mathrm{i}(K-k) \ell} \mathrm{e}^{\mathrm{i} k x}-\frac{4 \mathrm{i} V_{0}}{K^{2}-k^{2}} \mathrm{e}^{\mathrm{i} K x} .
$$

The third integral becomes

$$
\begin{gathered}
-2 V_{0} \int_{-b}^{b} \mathrm{e}^{\mathrm{i} K(x+\xi)} \int_{0}^{\sqrt{b^{2}-\xi^{2}}} H_{0}\left(k \sqrt{\xi^{2}+Y^{2}}\right) \mathrm{d} Y \mathrm{~d} \xi=-V_{0} \mathrm{e}^{\mathrm{i} K x} \int_{0}^{2 \pi} \int_{0}^{b} \mathrm{e}^{\mathrm{i} K r \cos \theta} H_{0}(k r) r \mathrm{~d} r \mathrm{~d} \theta \\
=-2 \pi V_{0} \mathrm{e}^{\mathrm{i} K x} \int_{0}^{b} J_{0}(K r) H_{0}(k r) r \mathrm{~d} r=V_{0} \mathrm{e}^{\mathrm{i} K x}\left\{\frac{4 \mathrm{i}}{K^{2}-k^{2}}-\frac{2 \pi \mathcal{N}_{0}(K b)}{K^{2}-k^{2}}\right\},
\end{gathered}
$$

where $\mathcal{N}_{0}(K b)=K b H_{0}(k b) J_{1}(K b)-k b H_{1}(k b) J_{0}(K b)$. Using these results in (37), we obtain

$$
V_{0} \mathrm{e}^{\mathrm{i} K x}-\mathrm{e}^{\mathrm{i} k x} \mathcal{A} \mathrm{e}^{\mathrm{i} K x}+\mathcal{B} \mathrm{e}^{\mathrm{i} k x} \quad \text { for } x>\ell+b,
$$

where

$$
\mathcal{A}=\frac{2 \pi g n_{0} V_{0}}{k^{2}-K^{2}} \mathcal{N}_{0}(K b), \quad \mathcal{B}=\frac{2 g n_{0}}{k} \int_{0}^{\ell} V(t) \mathrm{e}^{-\mathrm{i} k t} \mathrm{~d} t+\frac{2 \mathrm{i} g n_{0} V_{0}}{k(K-k)} \mathrm{e}^{\mathrm{i}(K-k) \ell}
$$


For a solution, we must have $\mathcal{A}=V_{0}$, and so

$$
K^{2}=k^{2}-2 \pi g n_{0} \mathcal{N}_{0}(K b),
$$

which is a nonlinear equation for $K$. We have $\mathcal{N}_{0}(K b) \rightarrow 2 \mathrm{i} / \pi$ as $b \rightarrow 0$ so that, in this limit, we recover Foldy's formula, (2).

Let us solve (38) for small $n_{0}$. (Alternatively, we could use the dimensionless area fraction $\phi \equiv \pi a^{2} n_{0}$.) Begin by writing

$$
K^{2}=k^{2}+\delta_{1} n_{0}+\delta_{2} n_{0}^{2}+\cdots,
$$

where $\delta_{1}$ and $\delta_{2}$ are to be found. It follows from (39) that $K=k+\frac{1}{2} \delta_{1} n_{0} / k+O\left(n_{0}^{2}\right)$ and then

$$
\mathcal{N}_{0}(K b)=\mathcal{N}_{0}(k b)+(K b-k b) \mathcal{N}_{0}^{\prime}(k b)+\cdots=2 \mathrm{i} / \pi+\frac{1}{2} b^{2} \delta_{1} d_{0}(k b) n_{0}+O\left(n_{0}^{2}\right),
$$

where $d_{0}(x)=J_{0}(x) H_{0}(x)+J_{1}(x) H_{1}(x)$. When this approximation for $\mathcal{N}_{0}(K b)$ is used in (38), we obtain

$$
K^{2}=k^{2}-4 \mathrm{i} g n_{0}-\pi b^{2} g \delta_{1} d_{0}(k b) n_{0}^{2} .
$$

Comparison of this formula with (39) gives $\delta_{1}=-4 \mathrm{i} g$ (as expected) and $\delta_{2}=4 \pi \mathrm{i}(g b)^{2} d_{0}(k b)$, so that we obtain the approximation

$$
K^{2}=k^{2}-4 \mathrm{i} g n_{0}+4 \pi \mathrm{i}\left(g b n_{0}\right)^{2} d_{0}(k b) .
$$

Note that the second-order term in (40) vanishes as $k b \rightarrow 0$. For more comments on (38) and (40), see Section 4.3.

\section{Finite-SIZE EFFECTS}

The theory described above relies on the assumption of isotropy. Here, we use a more complete theory. We start with an exact theory (due to Záviška) for acoustic scattering by $N$ identical circular cylinders of radius $a$; for details and references, see $[13$, p. 173] or $[16, \S 4.5]$. The cylinders can be soft, hard or penetrable. Then (in Section 3.2), we form averaged equations, and we invoke the QCA. This leads to an infinite homogeneous system of linear algebraic equations from which the effective wavenumber, $K$, is to be determined. An approximate solution for $K$ is found in Section 3.3, correct to $O\left(n_{0}^{2}\right)$.

\subsection{A finite array of identical circular cylinders: exact theory}

We use polar coordinates $(r, \theta)$ centred at the origin and $\left(r_{j}, \theta_{j}\right)$, centred at $\mathbf{r}_{j}=\left(x_{j}, y_{j}\right)$, the centre of the $j$ th cylinder. The various parameters relating to the relative positions of the cylinders are shown in Figure 1.

Exterior to the cylinders the total field is $u=u_{\text {in }}+u_{\mathrm{sc}}$, where $\left(\nabla^{2}+k^{2}\right) u_{\mathrm{sc}}=0$ and $u_{\mathrm{sc}}$ satisfies the Sommerfeld radiation condition. In the interior of cylinder $j$, the field is $u_{j}$, where $\left(\nabla^{2}+k_{0}^{2}\right) u_{j}=0$.

A plane wave is incident on the cylinders. We can write

$$
u_{\text {in }}=\mathrm{e}^{\mathrm{i} k x}=I_{j} \mathrm{e}^{\mathrm{i} k r_{j} \cos \theta_{j}}=I_{j} \sum_{n=-\infty}^{\infty} \mathrm{i}^{n} \mathrm{e}^{\mathrm{i} n \theta_{j}} J_{n}\left(k r_{j}\right),
$$

where $I_{j}=\mathrm{e}^{\mathrm{i} k x_{j}}$ and $J_{n}$ is a Bessel function. We seek $u_{\mathrm{sc}}$ and $u_{j}$ in the form

$$
\begin{aligned}
u_{\mathrm{sc}} & =\sum_{j=1}^{N} \sum_{n=-\infty}^{\infty} A_{n}^{j} Z_{n} H_{n}\left(k r_{j}\right) \mathrm{e}^{\mathrm{i} n \theta_{j}} \\
u_{j} & =\sum_{n=-\infty}^{\infty} B_{n}^{j} J_{n}\left(k_{0} r_{j}\right) \mathrm{e}^{\mathrm{i} n \theta_{j}}
\end{aligned}
$$




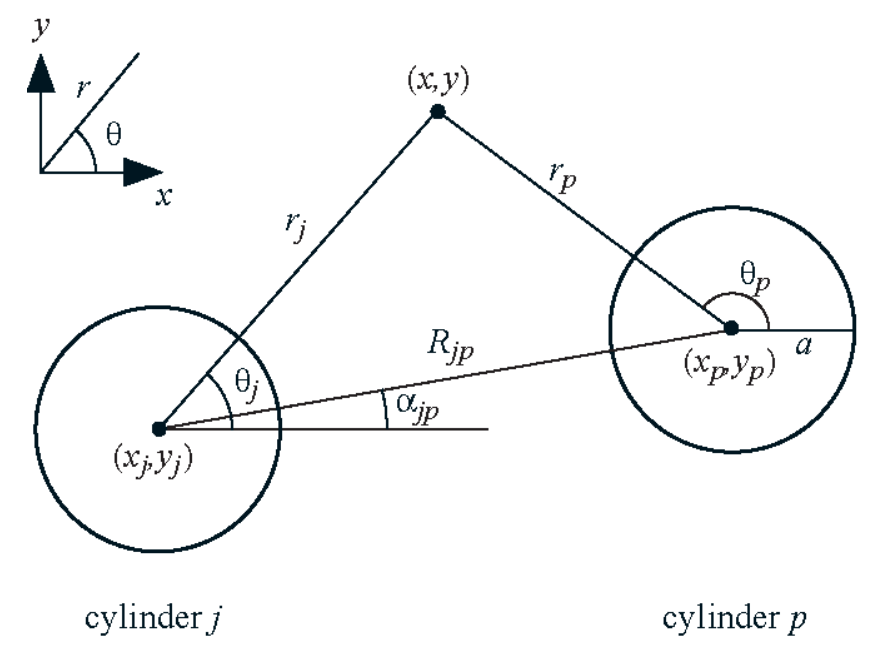

Figure 1. A view of two typical cylinders.

for some set of unknown complex coefficients $A_{n}^{j}$ and $B_{n}^{j}$. The factor

$$
Z_{n}=\frac{q J_{n}^{\prime}(k a) J_{n}\left(k_{0} a\right)-J_{n}(k a) J_{n}^{\prime}\left(k_{0} a\right)}{q H_{n}^{\prime}(k a) J_{n}\left(k_{0} a\right)-H_{n}(k a) J_{n}^{\prime}\left(k_{0} a\right)}=Z_{-n}
$$

has been introduced for later convenience. Here $k_{0}=\omega / c_{0}$ and $q=\rho_{0} c_{0} /(\rho c)$, where $\rho_{0}$ and $c_{0}$ are the density and sound speed, respectively, inside the cylinders. Note that we recover the sound-soft results in the limit $q \rightarrow 0$, whereas the limit $q \rightarrow \infty$ gives the sound-hard results. The boundary conditions on the cylinders are

$$
u=u_{s} \quad \text { and } \quad \frac{1}{\rho} \frac{\partial u}{\partial r_{s}}=\frac{1}{\rho_{0}} \frac{\partial u_{s}}{\partial r_{s}} \quad \text { on } \quad r_{s}=a, \quad s=1, \ldots, N .
$$

Using Graf's addition theorem for Bessel functions [16, Theorem 2.12], it can be shown that provided $r_{s}<R_{j s}$ for all $j$, we can write the field exterior to cylinder $s$ as

$$
\begin{aligned}
u\left(r_{s}, \theta_{s}\right)= & \sum_{n=-\infty}^{\infty}\left[I_{s} J_{n}\left(k r_{s}\right) \mathrm{i}^{n}+A_{n}^{s} Z_{n} H_{n}\left(k r_{s}\right)\right] \mathrm{e}^{\mathrm{i} n \theta_{s}} \\
& +\sum_{\substack{j=1 \\
j \neq s}}^{N} \sum_{n=-\infty}^{\infty} A_{n}^{j} Z_{n} \sum_{m=-\infty}^{\infty} J_{m}\left(k r_{s}\right) H_{n-m}\left(k R_{j s}\right) \mathrm{e}^{\mathrm{i} m \theta_{s}} \mathrm{e}^{\mathrm{i}(n-m) \alpha_{j s}} .
\end{aligned}
$$

The geometrical restriction implies that this expression is only valid if the point $\left(r_{s}, \theta_{s}\right)$ is closer to the centre of cylinder $s$ than the centres of any of the other cylinders. This is certainly true on the surface of cylinder $s$ and so (46) can be used to apply the body boundary conditions which leads, after using the orthogonality of the functions $\exp \left(\mathrm{i} m \theta_{s}\right), m \in \mathbb{Z}$, and eliminating the coefficients $B_{n}^{j}$, to the system of equations

$$
A_{m}^{s}+\sum_{\substack{j=1 \\
j \neq s}}^{N} \sum_{n=-\infty}^{\infty} A_{n}^{j} Z_{n} \mathrm{e}^{\mathrm{i}(n-m) \alpha_{j s}} H_{n-m}\left(k R_{j s}\right)=-I_{s} \mathrm{i}^{m}, \quad \begin{aligned}
& s=1,2, \ldots, N, \\
& m \in \mathbb{Z} .
\end{aligned}
$$


Note that the quantities $q, k_{0}$ and $a$ only enter the equations through the terms $Z_{n}$.

For a single cylinder at the origin, the scattered field is given by

$$
u_{\mathrm{sc}}(r, \theta)=-\sum_{n=-\infty}^{\infty} \mathrm{i}^{n} Z_{n} H_{n}(k r) \mathrm{e}^{\mathrm{i} n \theta}
$$

and then the far-field pattern, defined by (4), is given by

$$
f(\theta)=-\sum_{n=-\infty}^{\infty} Z_{n} \mathrm{e}^{\mathrm{i} n \theta}
$$

\subsection{Arrays of circular cylinders: averaged equations}

The above analysis applies to a specific configuration of scatterers. Now we follow Bose and Mal [2] and take ensemble averages. Thus, put $s=1$ in (47) and then take the conditional average, using (17), giving

$$
\left\langle A_{m}^{1}\right\rangle_{1}+n_{0} \frac{N-1}{N} \sum_{n=-\infty}^{\infty} Z_{n} \int_{B_{N}: R_{12}>b} H_{n-m}\left(k R_{21}\right) \mathrm{e}^{\mathrm{i}(n-m) \alpha_{21}}\left\langle A_{n}^{2}\right\rangle_{12} \mathrm{~d} V_{2}=-I_{1} \mathrm{i}^{m}, \quad m \in \mathbb{Z} .
$$

Next, let $N \rightarrow \infty$ so that $B_{N}$ becomes the half-space $x>0$, and invoke Lax's QCA, (25). This implies that $\left\langle A_{m}^{2}\right\rangle_{12}=\left\langle A_{m}^{2}\right\rangle_{2}$. We seek a solution to (50) in the form $\left\langle A_{m}^{s}\right\rangle_{s}=\mathrm{i}^{m} \Phi_{m}\left(x_{s}\right)$ so that

$$
\Phi_{m}\left(x_{1}\right)+n_{0} \sum_{n=-\infty}^{\infty} Z_{n}(-\mathrm{i})^{n-m} \int_{x_{2}>0, R_{12}>b} \psi_{n-m}\left(x_{21}, y_{21}\right) \Phi_{n}\left(x_{2}\right) \mathrm{d} x_{2} \mathrm{~d} y_{2}=-\mathrm{e}^{\mathrm{i} k x_{1}}, \quad m \in \mathbb{Z}
$$

where we have written $x_{21}=x_{2}-x_{1}$ and $y_{21}=y_{2}-y_{1}$, used $\alpha_{21}=\alpha_{12}-\pi$, and defined $\psi_{n}(X, Y)=H_{n}(k R) \mathrm{e}^{\mathrm{i} n \Theta}$ with $X=R \cos \Theta$ and $Y=R \sin \Theta$.

Proceeding as before, suppose that for sufficiently large $x$ (say $x>\ell$ ) we can write $\Phi_{m}(x)=F_{m} \mathrm{e}^{\mathrm{i} K x}$. We assume that $\operatorname{Im} K>0$ so that $\Phi_{m} \rightarrow 0$ as $x \rightarrow \infty$. Then if $x_{1}>\ell+b,(51)$ becomes

$$
F_{m} \mathrm{e}^{\mathrm{i} K x_{1}}+n_{0} \sum_{n=-\infty}^{\infty} Z_{n}(-\mathrm{i})^{n-m}\left\{\int_{0}^{\ell} \Phi_{n}\left(x_{2}\right) L_{n-m}\left(x_{21}\right) \mathrm{d} x_{2}+F_{n} \mathrm{e}^{\mathrm{i} K x_{1}} M_{n-m}\right\}=-\mathrm{e}^{\mathrm{i} k x_{1}}, \quad m \in \mathbb{Z},
$$

where

$$
\begin{aligned}
L_{n}(X) & =\int_{-\infty}^{\infty} \psi_{n}(X, Y) \mathrm{d} Y, \\
M_{n} & =\int_{x_{2}>\ell, R_{12}>b} \psi_{n}\left(x_{21}, y_{21}\right) \mathrm{e}^{\mathrm{i} K x_{21}} \mathrm{~d} x_{2} \mathrm{~d} y_{2} .
\end{aligned}
$$

The integrals $L_{n}$ and $M_{n}$ can be evaluated exactly [11]; the system (52) becomes

$$
\mathcal{A}_{m} \mathrm{e}^{\mathrm{i} K x}+\mathcal{B} \mathrm{e}^{\mathrm{i} k x}=-\mathrm{e}^{\mathrm{i} k x}, \quad x>\ell+b, \quad m \in \mathbb{Z},
$$

where

$$
\begin{aligned}
\mathcal{A}_{m} & =F_{m}+\frac{2 n_{0} \pi}{k^{2}-K^{2}} \sum_{n=-\infty}^{\infty} F_{n} Z_{n} \mathcal{N}_{n-m}(K b), \\
\mathcal{B} & =\frac{2 n_{0}}{k} \sum_{n=-\infty}^{\infty} Z_{n}\left\{\int_{0}^{\ell} \Phi_{n}(t) \mathrm{e}^{-\mathrm{i} k t} \mathrm{~d} t+\frac{\mathrm{i} F_{n}}{K-k} \mathrm{e}^{\mathrm{i}(K-k) \ell}\right\},
\end{aligned}
$$


and

$$
\mathcal{N}_{n}(K b)=k b H_{n}^{\prime}(k b) J_{n}(K b)-K b H_{n}(k b) J_{n}^{\prime}(K b) .
$$

In particular, note that $\mathcal{N}_{0}$ appeared in Section 2.3 during our analysis of Lax's integral equation.

From (55), we immediately obtain $\mathcal{B}=-1$ and $\mathcal{A}_{m}=0$ for all $m$; the second of these, namely

$$
F_{m}+\frac{2 n_{0} \pi}{k^{2}-K^{2}} \sum_{n=-\infty}^{\infty} F_{n} Z_{n} \mathcal{N}_{n-m}(K b)=0, \quad m \in \mathbb{Z}
$$

is of most interest to us. It is an infinite homogeneous system of linear algebraic equations for $F_{m}, m \in \mathbb{Z}$. The existence of a non-trivial solution to (57) determines $K$.

Equation (57) is the same as [2, eqn (33)] (with the choice (17)). However, the derivation of (57) given here has some advantages over that given by Bose and Mal [2]. First, we do not invoke "the so-called "extinction theorem" of Lax; this is described in [10, Sec. VI]. Roughly speaking, this "theorem" asserts that one may simply delete the incident field when calculating the effective wavenumber, in the limit $N \rightarrow \infty$. Along with this come some divergent integrals; for example, the integrals in the unnumbered equation between equations (32) and (33) in [2] are divergent, because $\mathrm{e}^{\mathrm{i} K x}$ is exponentially large as $x \rightarrow-\infty$. In fact, we can say that our analysis proves Lax's theorem in our particular case.

Second, when dealing with a half-space containing scatterers, we know from the work of Lloyd and Berry [14] that the boundary of the half-space can cause difficulties. Here, we give a proper treatment of this boundary. In particular, we do not assume that all fields are proportional to $\mathrm{e}^{\mathrm{i} K x}$ everywhere inside the half-space, $x>0$, but only in $x>\ell$, away from the boundary: the width of the boundary layer, $\ell$, is not specified, and need not be specified if one only wants to calculate $K$.

A more recent analysis in [20] permits non-circular and non-identical cylinders but assumes that the effective field is proportional to $\mathrm{e}^{\mathrm{i} K x}$ for all $x>0$.

\subsection{Approximate determination of $K$ for small $n_{0}$}

The only approximation made in the derivation of (57) is the QCA as closure assumption. This is expected to be valid for small values of the scatterer concentration $(\phi \ll 1)$. We now assume (as in Section 2.3$)$ that $n_{0} / k^{2}$ is also small and write $K^{2}=k^{2}+\delta_{1} n_{0}+\delta_{2} n_{0}^{2}+\ldots$. We then have

$$
\mathcal{N}_{n}(K b)=2 \mathrm{i} / \pi+\frac{1}{2} b^{2} \delta_{1} d_{n}(k b) n_{0}+O\left(n_{0}^{2}\right)
$$

where

$$
d_{n}(x)=J_{n}^{\prime}(x) H_{n}^{\prime}(x)+\left[1-(n / x)^{2}\right] J_{n}(x) H_{n}(x)
$$

and so

$$
\frac{\mathcal{N}_{n}(K b)}{k^{2}-K^{2}}=-\frac{2 \mathrm{i}}{\pi \delta_{1} n_{0}}-\frac{b^{2} d_{n}(k b)}{2}+\frac{2 \mathrm{i} \delta_{2}}{\pi \delta_{1}^{2}}+O\left(n_{0}\right) .
$$

If $(60)$ is substituted in $(57)$ and $O\left(n_{0}^{2}\right)$ terms neglected we get

$$
F_{m}=\frac{4 \mathrm{i}}{\delta_{1}} \sum_{n=-\infty}^{\infty} Z_{n} F_{n}+n_{0} \sum_{n=-\infty}^{\infty} Z_{n} F_{n}\left(\pi b^{2} d_{n-m}(k b)-\frac{4 \mathrm{i} \delta_{2}}{\delta_{1}^{2}}\right), \quad m \in \mathbb{Z}
$$

At leading order this gives

$$
F_{m}=\frac{4 \mathrm{i}}{\delta_{1}} \sum_{n=-\infty}^{\infty} Z_{n} F_{n}, \quad m \in \mathbb{Z}
$$


which implies that all the $F_{m}$ are equal. If we write $F_{m}=F,(62)$ becomes

$$
\delta_{1}=4 \mathrm{i} \sum_{s=-\infty}^{\infty} Z_{s}=-4 \mathrm{i} f(0)
$$

where $f$ is the far-field pattern, given by (49).

Returning to (61), we now put $F_{m}=F+n_{0} q_{m}$, and then the $O\left(n_{0}\right)$ terms give

$$
q_{m}=-\frac{1}{f(0)} \sum_{n=-\infty}^{\infty} Z_{n} q_{n}+\pi b^{2} F \sum_{n=-\infty}^{\infty} Z_{n} d_{n-m}(k b)-\frac{\mathrm{i} F \delta_{2}}{4 f(0)}, \quad m \in \mathbb{Z} .
$$

It follows that $q_{m}-\pi b^{2} F \sum_{n=-\infty}^{\infty} Z_{n} d_{n-m}$ must be independent of $m$, call it $Q$ :

$$
Q=-\frac{1}{f(0)} \sum_{n=-\infty}^{\infty} Z_{n} q_{n}-\frac{\mathrm{i} F \delta_{2}}{4 f(0)}=-\frac{1}{f(0)} \sum_{n=-\infty}^{\infty} Z_{n}\left(Q+F \pi b^{2} \sum_{s=-\infty}^{\infty} Z_{s} d_{s-n}(k b)\right)-\frac{\mathrm{i} F \delta_{2}}{4 f(0)} .
$$

Hence

$$
\delta_{2}=4 \pi \mathrm{i} b^{2} \sum_{n=-\infty}^{\infty} \sum_{s=-\infty}^{\infty} Z_{n} Z_{s} d_{s-n}(k b)
$$

and so we obtain the approximation

$$
K^{2}=k^{2}-4 \mathrm{i} n_{0} f(0)+4 \pi \mathrm{i} b^{2} n_{0}^{2} \sum_{n=-\infty}^{\infty} \sum_{s=-\infty}^{\infty} Z_{n} Z_{s} d_{s-n}(k b)+\ldots
$$

For isotropic point scatterers, we have $\left|Z_{0}\right| \gg\left|Z_{n}\right|$ for all $n \neq 0$ and $g=-Z_{0}$, so that (66) reduces to (40) in this limit.

So far we have not made any assumptions about the size of $k a$ or $k b$ (though clearly $k b \geq 2 k a$ ). Now we will assume that $k b$ is small. In the limit $x \rightarrow 0$, we have $x^{2} d_{n}(x) \sim 2 \mathrm{i}|n| / \pi$. Hence as $k b \rightarrow 0$,

$$
\delta_{2} \sim-\frac{8}{k^{2}} \sum_{n=-\infty}^{\infty} \sum_{s=-\infty}^{\infty}|s-n| Z_{n} Z_{s}
$$

Now

$$
[f(\theta)]^{2}=\sum_{n=-\infty}^{\infty} \sum_{s=-\infty}^{\infty} Z_{n} Z_{s} \mathrm{e}^{\mathrm{i}(n+s) \theta}=\sum_{n=-\infty}^{\infty} \sum_{s=-\infty}^{\infty} Z_{n} Z_{s} \cos (n-s) \theta
$$

since $Z_{n}=Z_{-n}$. Thus

$$
\frac{\mathrm{d}}{\mathrm{d} \theta}[f(\theta)]^{2}=-\sum_{n=-\infty}^{\infty} \sum_{s=-\infty}^{\infty}(n-s) Z_{n} Z_{s} \sin (n-s) \theta .
$$

Also

$$
\int_{0}^{\pi} \cot \frac{1}{2} \theta \sin m \theta \mathrm{d} \theta=\pi \operatorname{sgn}(m),
$$

see [7, eqn 3.612(7)]. Thus, setting $k b=0$ gives the formula (11).

The effect of using (18) for the pair-correlation function has been analysed in [11, §IV.D]; it does not change (11). Also, following [14], it can be shown that a poor choice for the pair-correlation function will lead to Twersky's (erroneous) formula, (8); see [11, §IV.E]. 


\section{WEAK SCATTERING}

The analysis in Section 3 gives results for identical circular cylinders of any composition, hard or soft, penetrable or not. We are going to specialise these results to weak scattering, which means penetrable cylinders with $\rho=\rho_{0}$ and $k \simeq k_{0}$. We will then be able to make comparisons with results obtained by solving the Lippmann-Schwinger integral equation by iteration.

Consider one circle of radius $a$, centred at the origin. We have (see Section 3.1) $\left(\nabla^{2}+k^{2}\right) u_{\mathrm{sc}}=0$ for $r>a$ and $\left(\nabla^{2}+k_{0}^{2}\right) u_{0}=0$ for $r<a$. Assume for simplicity that $k$ and $k_{0}$ are both real. The interface conditions are $u=u_{0}$ and $\partial u / \partial r=\partial u_{0} / \partial r$ on $r=a$ where $u=u_{\text {in }}+u_{\mathrm{sc}}$. Then, $u_{\mathrm{sc}}$ is given by (48) with $Z_{n}$ defined by (44); as $\rho=\rho_{0}$ and $k$ and $k_{0}$ are real, we have

$$
Z_{n}=\left[\operatorname{Re} \Delta_{n}\right] / \Delta_{n}=Z_{-n}
$$

with

$$
\Delta_{n}=H_{n}^{\prime}(k a) J_{n}\left(k_{0} a\right)-\left(k_{0} / k\right) J_{n}^{\prime}\left(k_{0} a\right) H_{n}(k a) .
$$

The interior field is given by

$$
u_{0}(r, \theta)=\sum_{n=-\infty}^{\infty} B_{n} J_{n}\left(k_{0} r\right) \mathrm{e}^{\mathrm{i} n \theta} \quad \text { with } \quad B_{n}=\frac{2 \mathrm{i}^{n+1}}{\pi k a \Delta_{n}}
$$

The far-field pattern, $f(\theta)$, is defined by (4); it is given by (49) in terms of $Z_{n}$.

\subsection{Approximation for small $m_{0}$}

For weak scattering, $k_{0}$ is close to $k$. To quantify this, we define the scattering strength, $m_{0}$, by

$$
m_{0}=1-\left(k_{0} / k\right)^{2} .
$$

Then, for weak scattering, we approximate the exact solution, assuming that $m_{0}$ is small. From (74), we obtain

$$
k_{0} / k \simeq 1-\frac{1}{2} m_{0}-\frac{1}{8} m_{0}^{2}
$$

and

$$
F\left(k_{0} a\right) \simeq F(k a)-\frac{1}{2} m_{0} k a F^{\prime}(k a)-\frac{1}{8} m_{0}^{2} k a\left\{F^{\prime}(k a)-k a F^{\prime \prime}(k a)\right\}
$$

for any smooth function $F$. Then, some calculation gives

$$
\Delta_{n} \simeq 2 \mathrm{i} /(\pi k a)-\left(m_{0} / 2\right) k a d_{n}(k a)+\left(m_{0}^{2} / 8\right) U_{n}
$$

where $d_{n}(x)$ is defined by (59),

$$
\begin{aligned}
U_{n} & =k a\left(k a J_{n}^{\prime \prime}-J_{n}^{\prime}\right) H_{n}^{\prime}+\left(J_{n}^{\prime}-k a\left[J_{n}^{\prime \prime}+k a J_{n}^{\prime \prime \prime}\right]\right) H_{n} \\
& =2 k a\left[J_{n}(k a) H_{n}(k a)-d_{n}(k a)-\mathrm{i} / \pi\right]+2 \mathrm{i} n^{2} /(\pi k a)
\end{aligned}
$$

and we have used

$$
H_{n}^{\prime}(x) J_{n}(x)-J_{n}^{\prime}(x) H_{n}(x)=2 \mathrm{i} /(\pi x) .
$$

Then, from (77), the numerator in (71) is given approximately by

$$
\operatorname{Re} \Delta_{n} \simeq-\left(m_{0} / 2\right) k a \mathcal{J}_{n}(k a)+\left(m_{0}^{2} / 8\right) S_{n}
$$


where

$$
\begin{aligned}
\mathcal{J}_{n}(k a) & =\operatorname{Re} d_{n}(k a)=J_{n}^{2}(k a)-J_{n-1}(k a) J_{n+1}(k a), \\
S_{n} & =\operatorname{Re} U_{n}=2 k a J_{n-1}(k a) J_{n+1}(k a) .
\end{aligned}
$$

Hence, we obtain the following approximation for $Z_{n}$ :

$$
Z_{n} \simeq \frac{\pi}{4} m_{0} \mathrm{i}(k a)^{2} \mathcal{J}_{n}(k a)-\frac{\pi}{16} m_{0}^{2} k a\left\{\mathrm{i} S_{n}-\pi(k a)^{3} \mathcal{J}_{n}(k a) d_{n}(k a)\right\} .
$$

Similarly, for the interior field, $u_{0},(73)$ gives

$$
\mathrm{i}^{-n} B_{n} \simeq 1-\frac{\mathrm{i} m_{0}}{4} \mu_{n}+\frac{m_{0}^{2}}{16}\left(\pi \mathrm{i} k a U_{n}-\mu_{n}^{2}\right) \quad \text { with } \quad \mu_{n}=\pi(k a)^{2} d_{n}(k a) .
$$

Also, using (76),

$$
J_{n}\left(k_{0} r\right) \simeq J_{n}(k r)-\left(m_{0} / 2\right) k r J_{n}^{\prime}(k r)+\left(m_{0}^{2} / 8\right)\left\{\left[n^{2}-(k r)^{2}\right] J_{n}(k r)-2 k r J_{n}^{\prime}(k r)\right\},
$$

and then (73) gives

$$
u_{0}(r, \theta) \simeq \mathrm{e}^{\mathrm{i} k x}-\frac{\mathrm{i} m_{0}}{4} \sum_{n=-\infty}^{\infty} \mathrm{i}^{n} u_{n}^{(1)} \mathrm{e}^{\mathrm{i} n \theta}+\frac{m_{0}^{2}}{16} \sum_{n=-\infty}^{\infty} \mathrm{i}^{n} u_{n}^{(2)} \mathrm{e}^{\mathrm{i} n \theta}
$$

where

$$
\begin{aligned}
& u_{n}^{(1)}=\mu_{n} J_{n}(k r)-2 \mathrm{i} k r J_{n}^{\prime}(k r), \\
& u_{n}^{(2)}=\left\{2\left[n^{2}-(k r)^{2}\right]+\pi \mathrm{i} k a U_{n}-\mu_{n}^{2}\right\} J_{n}(k r)+2\left(\mathrm{i} \mu_{n}-2\right) k r J_{n}^{\prime}(k r) .
\end{aligned}
$$

\subsection{Application to Foldy theory}

Foldy's theory predicts that $\langle u\rangle=A \mathrm{e}^{\mathrm{i} K x}$, where $K$ is given by (5) and $A$ is given by (7). Using (49), (5) gives

$$
K^{2}-k^{2}=4 \mathrm{i} n_{0} \sum_{n=-\infty}^{\infty} Z_{n} \simeq-m_{0} n_{0} \pi(k a)^{2} \sum_{n=-\infty}^{\infty} \mathcal{J}_{n}(k a)=-m_{0} n_{0} \pi(k a)^{2},
$$

to first order in $m_{0}$. Here, we have used

$$
\begin{aligned}
& \sum_{n=-\infty}^{\infty} \mathcal{J}_{n}=\sum_{n=-\infty}^{\infty} J_{n}^{2}-\sum_{n=-\infty}^{\infty} J_{n} J_{n+2}=1, \\
& \sum_{n=-\infty}^{\infty} J_{n}^{2}(x)=1, \\
& \sum_{n=-\infty}^{\infty} J_{n}(x) J_{n+m}(x)=0 \quad \text { for } m= \pm 1, \pm 2, \ldots
\end{aligned}
$$

Also, from (7), we obtain $A \simeq 1+\frac{1}{4} \pi m_{0} n_{0} a^{2}$.

For an approximation to second order in $m_{0}$, use (82). The term in $S_{n}$ does not contribute to $f(0)$ : $\sum_{n=-\infty}^{\infty} S_{n}=0$. To see this, use (81) and (87). Thus, we obtain

$$
f(0)=-\frac{1}{4} \mathrm{i} m_{0} \pi(k a)^{2}+\frac{1}{4} \mathrm{i} m_{0}^{2} \pi^{2}(k a)^{4} \mathcal{H}(k a),
$$


where

$$
\mathcal{H}(k a)=\frac{\mathrm{i}}{4} \sum_{n=-\infty}^{\infty} \mathcal{J}_{n}(k a) d_{n}(k a)
$$

Then (5) gives

$$
K^{2}-k^{2} \simeq-m_{0} n_{0} \pi(k a)^{2}+m_{0}^{2} n_{0} \pi^{2}(k a)^{4} \mathcal{H}(k a)
$$

and (7) gives

$$
A \simeq 1+\frac{1}{4} m_{0} n_{0} \pi a^{2}-\frac{1}{4} m_{0}^{2} n_{0} \pi^{2} k^{2} a^{4} \mathcal{H}(k a) .
$$

\subsection{Application to Linton-Martin theory}

According to Linton and Martin [11], the second-order correction in (3) is given by (65). As a special case of (65), consider isotropic scattering, meaning that $Z_{n}=0$ when $n \neq 0$, so that $f(\theta)=-Z_{0}$. Then, we obtain the estimate (40) with $g=f(0)$ therein. This estimate is also [4, eqn (5)] when $k_{\text {eff }}$ is replaced by $k_{0}$ in its right-hand side. (In fact, [4, eqn (5)] is equivalent to (38).)

From (82), the first-order approximation to $Z_{n}$ is given by

$$
Z_{n} \simeq\left(m_{0} / 4\right) \pi \mathrm{i}(k a)^{2} \mathcal{J}_{n}(k a),
$$

whence (65) gives

$$
\delta_{2} \simeq m_{0}^{2} \frac{\pi^{3}}{4 \mathrm{i}} b^{2}(k a)^{4} \sum_{n=-\infty}^{\infty} \sum_{m=-\infty}^{\infty} \mathcal{J}_{n}(k a) \mathcal{J}_{m}(k a) d_{n-m}(k b) .
$$

Thus, there are no contributions to $K^{2}$ that are proportional to $n_{0}^{2} m_{0}$.

\section{LIPPMANN-SCHWINGER EQUATION}

In two dimensions, the Lippmann-Schwinger integral equation is [3]

$$
u(\mathbf{r})=u_{\text {in }}(\mathbf{r})-k^{2} \int G_{0}\left(\mathbf{r}, \mathbf{r}^{\prime}\right) m\left(\mathbf{r}^{\prime}\right) u\left(\mathbf{r}^{\prime}\right) \mathrm{d} V^{\prime}
$$

where $u$ is the total field and $G_{0}\left(\mathbf{r}, \mathbf{r}^{\prime}\right)=(\mathrm{i} / 4) H_{0}^{(1)}\left(k\left|\mathbf{r}-\mathbf{r}^{\prime}\right|\right)$. The governing partial differential equation is

$$
\nabla^{2} u+k^{2} u=k^{2} m(\mathbf{r}) u
$$

with $m \equiv 0$ outside the scatterers.

It is known that (94) is always uniquely solvable. Moreover, the solution can be constructed by iterating the integral equation, under certain circumstances. We do not investigate these circumstances here. We simply accept the second iteration, giving

$$
\begin{aligned}
u(\mathbf{r}) \simeq & u_{\text {in }}(\mathbf{r})-k^{2} \int G_{0}\left(\mathbf{r}, \mathbf{r}^{\prime}\right) m\left(\mathbf{r}^{\prime}\right) u_{\text {in }}\left(\mathbf{r}^{\prime}\right) \mathrm{d} V^{\prime} \\
& +k^{4} \int G_{0}\left(\mathbf{r}, \mathbf{r}^{\prime}\right) m\left(\mathbf{r}^{\prime}\right) \int G_{0}\left(\mathbf{r}^{\prime}, \mathbf{r}^{\prime \prime}\right) m\left(\mathbf{r}^{\prime \prime}\right) u_{\text {in }}\left(\mathbf{r}^{\prime \prime}\right) \mathrm{d} V^{\prime \prime} \mathrm{d} V^{\prime} .
\end{aligned}
$$

We assume that the scatterers are $D_{i}$, centred at $\mathbf{r}_{i}, i=1,2, \ldots, N$. Each scatterer is a circular disc of radius $a$ with constant strength $m_{0}$; when (74) is combined with (95), we see that $\left(\nabla^{2}+k_{0}^{2}\right) u=0$ holds inside each disc. Thus

$$
\left(\nabla^{2}+k^{2}\right) u=k^{2} m_{0} u(\mathbf{r}) \sum_{i=1}^{N} \chi_{i}(\mathbf{r}),
$$


where $\chi_{i}$ is the characteristic function for $D_{i}: \chi_{i}(\mathbf{r})=1$ when $\mathbf{r} \in D_{i}$ and $\chi_{i}(\mathbf{r})=0$ when $\mathbf{r} \notin D_{i}$. The approximation (96) becomes

$$
\begin{aligned}
u(\mathbf{r}) \simeq & u_{\text {in }}(\mathbf{r})-k^{2} m_{0} \sum_{i=1}^{N} \int_{D_{i}} G_{0}\left(\mathbf{r}, \mathbf{r}^{\prime}\right) u_{\text {in }}\left(\mathbf{r}^{\prime}\right) \mathrm{d} V^{\prime} \\
& +k^{4} m_{0}^{2} \sum_{i=1}^{N} \sum_{j=1}^{N} \int_{D_{i}} G_{0}\left(\mathbf{r}, \mathbf{r}^{\prime}\right) \int_{D_{j}} G_{0}\left(\mathbf{r}^{\prime}, \mathbf{r}^{\prime \prime}\right) u_{\text {in }}\left(\mathbf{r}^{\prime \prime}\right) \mathrm{d} V^{\prime \prime} \mathrm{d} V^{\prime} .
\end{aligned}
$$

We shall return to the formula (98) in Section 7, but first we consider scattering by one cylinder.

\section{SCATtERING By ONE CYLINDER: ITERATIVE SOLUTION}

Let us specialise the second-order solution (98) to a single cylinder. We have

$$
u(\mathbf{r}) \simeq u_{\text {in }}(\mathbf{r})+m_{0} I_{1}(\mathbf{r})+m_{0}^{2} I_{2}(\mathbf{r})
$$

where

$$
\begin{aligned}
& I_{1}(\mathbf{r})=-k^{2} \int_{D_{0}} G_{0}\left(\mathbf{r}, \mathbf{r}^{\prime}\right) u_{\text {in }}\left(\mathbf{r}^{\prime}\right) \mathrm{d} V^{\prime}, \\
& I_{2}(\mathbf{r})=-k^{2} \int_{D_{0}} G_{0}\left(\mathbf{r}, \mathbf{r}^{\prime}\right) I_{1}\left(\mathbf{r}^{\prime}\right) \mathrm{d} V^{\prime},
\end{aligned}
$$

and $D_{0}$ is the circular disc of radius $a$, centred at the origin. In this section, we will evaluate $I_{1}$ and $I_{2}$. These quantities will be needed when we consider scattering by many circles (in Section 7). Also, as a check on our calculations, we verify that we recover the small- $m_{0}$ approximations to the exact solution given in Section 4.1.

\subsection{Evaluation of $I_{1}$}

With $\mathbf{r}=(r, \theta)$ and $\mathbf{r}^{\prime}=\left(r^{\prime}, \theta^{\prime}\right)$, we use (41) in (100) to give

$$
I_{1}(r, \theta)=-\sum_{n=-\infty}^{\infty} \mathrm{i}^{n} L_{n}(r, \theta)
$$

where

$$
L_{n}(r, \theta)=k^{2} \int_{0}^{a} \int_{0}^{2 \pi} G_{0}\left(\mathbf{r}, \mathbf{r}^{\prime}\right) J_{n}\left(k r^{\prime}\right) \mathrm{e}^{\mathrm{i} n \theta^{\prime}} r^{\prime} \mathrm{d} \theta^{\prime} \mathrm{d} r^{\prime} .
$$

We give separate evaluations of $L_{n}(r, \theta)$ for $r>a$ and $r<a$.

\subsubsection{Exterior field}

For $r>a$, we use [16, p. 40]

$$
G_{0}\left(\mathbf{r}, \mathbf{r}^{\prime}\right)=\frac{\mathrm{i}}{4} \sum_{n=-\infty}^{\infty} H_{n}(k r) J_{n}\left(k r^{\prime}\right) \mathrm{e}^{\mathrm{i} n\left(\theta-\theta^{\prime}\right)}, \quad r>r^{\prime},
$$

in (103). This gives

with

$$
L_{n}(r, \theta)=C_{n}^{(1)} H_{n}(k r) \mathrm{e}^{\mathrm{i} n \theta},
$$

$$
C_{n}^{(1)}=\frac{\pi \mathrm{i}}{2} k^{2} \int_{0}^{a} J_{n}^{2}\left(k r^{\prime}\right) r^{\prime} \mathrm{d} r^{\prime}=\frac{\pi \mathrm{i}}{4}(k a)^{2} \mathcal{J}_{n}(k a),
$$


where $\mathcal{J}_{n}$ is defined by (80). Here, we have used the standard integral

$$
\int w_{n}(k r) W_{n}(k r) r \mathrm{~d} r \frac{r^{2}}{4}\left\{2 w_{n}(k r) W_{n}(k r)-w_{n+1}(k r) W_{n-1}(k r)-w_{n-1}(k r) W_{n+1}(k r)\right\},
$$

where $w_{n}$ and $W_{n}$ are any two Bessel functions. In particular, we note that

$$
\frac{2}{a^{2}} \int_{0}^{a} J_{n}^{2}(k r) r \mathrm{~d} r=\mathcal{J}_{n}(k a)
$$

a result that will be useful later. The result (106) agrees with the exact solution, see (92).

\subsubsection{Interior field}

For $r<a$, the calculation is more complicated. We obtain

$$
L_{n}(r, \theta)=(\pi \mathrm{i} / 8) \Lambda_{n}^{(1)}(k r) \mathrm{e}^{\mathrm{i} n \theta}, \quad r<a,
$$

with

$$
\begin{gathered}
\Lambda_{n}^{(1)}(k r) 4 k^{2} H_{n}(k r) \int_{0}^{r} J_{n}^{2}\left(k r^{\prime}\right) r^{\prime} \mathrm{d} r^{\prime}+4 k^{2} J_{n}(k r) \int_{r}^{a} H_{n}\left(k r^{\prime}\right) J_{n}\left(k r^{\prime}\right) r^{\prime} \mathrm{d} r^{\prime} \\
=-(4 \mathrm{i} / \pi) k r J_{n}^{\prime}(k r)+2(k a)^{2} J_{n}(k r) d_{n}(k a),
\end{gathered}
$$

where $d_{n}$ is defined by (59). Combining (99), (102), (109) and (111), we find agreement with the first-order approximation of the exact solution, (83).

\subsection{Evaluation of $I_{2}$}

As $r^{\prime}<a$ in (101), we use (109) in (102) to give

$$
I_{2}(\mathbf{r})=\frac{\pi \mathrm{i}}{8} k^{2} \sum_{m=-\infty}^{\infty} \mathrm{i}^{m} \int G_{0}\left(\mathbf{r}, \mathbf{r}^{\prime}\right) \Lambda_{m}^{(1)}\left(k r^{\prime}\right) \mathrm{e}^{\mathrm{i} m \theta^{\prime}} \mathrm{d} V^{\prime} .
$$

Again, the calculations depend on whether $r>a$ or $r<a$.

\subsubsection{Exterior field}

For $r>a$, we use (104). This gives

$$
I_{2}(r, \theta)=-\sum_{n=-\infty}^{\infty} \mathrm{i}^{n} C_{n}^{(2)} H_{n}(k r) \mathrm{e}^{\mathrm{i} n \theta},
$$

with

$$
\begin{aligned}
C_{n}^{(2)} & =\frac{\pi^{2} k^{2}}{16} \int_{0}^{a} J_{n}\left(k r^{\prime}\right) \Lambda_{n}^{(1)}\left(k r^{\prime}\right) r^{\prime} \mathrm{d} r^{\prime} \\
& =(\pi k a / 4)^{2}\left\{(k a)^{2} d_{n}(k a) \mathcal{J}_{n}(k a)-(2 \mathrm{i} / \pi) J_{n-1}(k a) J_{n+1}(k a)\right\}
\end{aligned}
$$

The result (114) agrees with the exact solution, see (82). 


\subsubsection{Interior field}

For $r<a$, we obtain

$$
I_{2}(r, \theta)=\left(\frac{\pi}{8 \mathrm{i}}\right)^{2} \sum_{n=-\infty}^{\infty} \mathrm{i}^{n} \Lambda_{n}^{(2)}(k r) \mathrm{e}^{\mathrm{i} n \theta}, \quad r<a,
$$

with

$$
\begin{gathered}
\Lambda_{n}^{(2)}(k r) 4 k^{2} H_{n}(k r) \int_{0}^{r} J_{n}\left(k r^{\prime}\right) \Lambda_{n}^{(1)}\left(k r^{\prime}\right) r^{\prime} \mathrm{d} r^{\prime}+4 k^{2} J_{n}(k r) \int_{r}^{a} H_{n}\left(k r^{\prime}\right) \Lambda_{n}^{(1)}\left(k r^{\prime}\right) r^{\prime} \mathrm{d} r^{\prime} \\
=2(k a)^{2} d_{n}(k a) \Lambda_{n}^{(1)}(k r)-8(\mathrm{i} / \pi) X_{n},
\end{gathered}
$$

using (111),

$$
\begin{aligned}
X_{n} & =2 k^{3} H_{n}(k r) \int_{0}^{r} J_{n}(k s) J_{n}^{\prime}(k s) s^{2} \mathrm{~d} s+2 k^{3} J_{n}(k r) \int_{r}^{a} H_{n}(k s) J_{n}^{\prime}(k s) s^{2} \mathrm{~d} s \\
& =(\mathrm{i} k r / \pi)\left\{J_{n-1}(k r)-J_{n+1}(k r)+k r J_{n}(k r)\right\}+\left(k a U_{n} / 2-n^{2} \mathrm{i} / \pi\right) J_{n}(k r)
\end{aligned}
$$

and $U_{n}$ is defined by (78). When $I_{2}$ is substituted in (99), we find complete agreement with the second-order approximation to the exact solution, (83).

\section{SCATTERING By $N$ CYLINDERS}

Let us return to the second-order solution for scattering by $N$ cylinders, given by (98). Introduce polar coordinates $(r, \theta)$ centred at the origin and $\left(r_{j}, \theta_{j}\right)$ centred at $\mathbf{r}_{j}=\left(x_{j}, y_{j}\right)$, the centre of the $j$ th scatterer.

\subsection{First order in $m_{0}$}

As in Section 6, using $u_{\text {in }}=\mathrm{e}^{\mathrm{i} k x}=\mathrm{e}^{\mathrm{i} k x_{j}} \mathrm{e}^{\mathrm{i} k r_{j} \cos \theta_{j}}$, we obtain

$$
-k^{2} \int_{D_{j}} G_{0}\left(\mathbf{r}, \mathbf{r}^{\prime}\right) u_{\text {in }}\left(\mathbf{r}^{\prime}\right) \mathrm{d} V^{\prime} \mathrm{e}^{\mathrm{i} k x_{j}} I_{1}\left(r_{j}, \theta_{j}\right),
$$

with $r_{j} \equiv\left|\mathbf{r}-\mathbf{r}_{j}\right|$, so that, to first order in $m_{0},(98)$ gives

$$
u(\mathbf{r}) \simeq \mathrm{e}^{\mathrm{i} k x}+m_{0} \sum_{j=1}^{N} \mathrm{e}^{\mathrm{i} k x_{j}} I_{1}\left(r_{j}, \theta_{j}\right) .
$$

Next, we calculate the ensemble average of $u,\langle u\rangle$. At this stage, we can assume that the scatterers are independent (uncorrelated); they are also indistinguishable. The result is

$$
\langle u(\mathbf{r})\rangle \mathrm{e}^{\mathrm{i} k x}+m_{0} n_{0} \int_{B_{N}} \mathrm{e}^{\mathrm{i} k x_{1}} I_{1}\left(r_{1}, \theta_{1}\right) \mathrm{d} x_{1} \mathrm{~d} y_{1},
$$

where $B_{N}$ is the region occupied by the $N$ circles.

We now let $N \rightarrow \infty$. One option would be for $B_{N}$ to become a slab, $0<x<L$, say. We prefer to let $B_{N}$ become the half-plane $x>0$, but then we have to take care with the convergence of various integrals as $x \rightarrow \infty$ : we replace $\mathrm{e}^{\mathrm{i} k x_{1}}$ by $\mathrm{e}^{\mathrm{i} \kappa x_{1}}$, with $\operatorname{Im} \kappa>0$, and then let $\kappa \rightarrow k$ at the end of the calculation.

Thus, in the limit $N \rightarrow \infty$, the integral in (120) consists of an integral over the circular disc $r_{1}<a$ $\left(r_{1}^{2}=\left(x_{1}-x\right)^{2}+y_{1}^{2}\right)$ plus an integral over the remainder of the half-plane $x_{1}>0$; we denote this region by disc $^{\prime}$. We can assume here that $x>a$ so that the disc does not meet the line $x_{1}=0$. 
Exact calculation gives [17]

$$
\begin{gathered}
\int_{r_{1}<a} \mathrm{e}^{\mathrm{i} k x_{1}} I_{1}\left(r_{1}, \theta_{1}\right) \mathrm{d} x_{1} \mathrm{~d} y_{1}=-\pi^{2} a^{2} \mathrm{e}^{\mathrm{i} k x}(k a)^{2} \mathcal{H}(k a), \\
\lim _{\kappa \rightarrow k} \int_{\text {disc' }^{\prime}} \mathrm{e}^{\mathrm{i} \kappa x_{1}} I_{1}\left(r_{1}, \theta_{1}\right) \mathrm{d} x_{1} \mathrm{~d} y_{1} \frac{\pi a^{2}}{4} \mathrm{e}^{\mathrm{i} k x}\left\{1-2 \mathrm{i} k x+4 \pi(k a)^{2} \mathcal{H}(k a)\right\},
\end{gathered}
$$

where $\mathcal{H}$ is defined by (89).

Digression on the evaluation of (122).

From (102) and (105), we have

$$
\begin{aligned}
& \int_{\text {disc' }^{\prime}} \mathrm{e}^{\mathrm{i} \kappa x_{1}} I_{1} \mathrm{~d} x_{1} \mathrm{~d} y_{1}=-\sum_{n=-\infty}^{\infty} \mathrm{i}^{n} C_{n}^{(1)} \int_{\text {disc' }^{\prime}} H_{n}\left(k r_{1}\right) \mathrm{e}^{\mathrm{i} n \theta_{1}} \mathrm{e}^{\mathrm{i} \kappa x_{1}} \mathrm{~d} x_{1} \mathrm{~d} y_{1} \\
& \frac{2 \mathrm{i}}{k} \sum_{n=-\infty}^{\infty} C_{n}^{(1)} \frac{(\kappa+k) \mathrm{e}^{\mathrm{i} k x}+\pi \mathrm{i} k \mathrm{e}^{\mathrm{i} \kappa x} \mathcal{N}_{n}(\kappa a)}{k^{2}-\kappa^{2}},
\end{aligned}
$$

where $\operatorname{Im} \kappa>0$ and

$$
\mathcal{N}_{n}(\kappa a)=k a H_{n}^{\prime}(k a) J_{n}(\kappa a)-\kappa a H_{n}(k a) J_{n}^{\prime}(\kappa a) .
$$

Here, we have evaluated the integral on the right-hand side of (123) as on p. 3419 of [11]; the result is exact when $x>a$. Recall that the region disc' consists of that part of the half-plane $x_{1}>0$ that is outside the circle $r_{1}^{2} \equiv\left(x_{1}-x\right)^{2}+y_{1}^{2}=a^{2}$. The method of evaluation in [11] uses Green's theorem to reduce the double integral to the sum of an integral along $x_{1}=0$ and an integral around the circle $r_{1}=a$. When $0<x<a$, this circle cuts the $y_{1}$-axis, and then (124) should be regarded as an approximation. More precisely, we have

$$
\int_{\text {disc' }^{\prime}} H_{n}\left(k r_{1}\right) \mathrm{e}^{\mathrm{i} n \theta_{1}} \mathrm{e}^{\mathrm{i} \kappa x_{1}} \mathrm{~d} x_{1} \mathrm{~d} y_{1}=\frac{2}{\mathrm{i} k}(-\mathrm{i})^{n} \frac{(\kappa+k) \mathrm{e}^{\mathrm{i} k x}+\pi \mathrm{i} k \mathrm{e}^{\mathrm{i} \kappa x} \mathcal{N}_{n}(\kappa a)}{k^{2}-\kappa^{2}}+E_{n}(x),
$$

where $E_{n}(x)=0$ for $x \geq a$,

$$
E_{n}(x)=\int_{\mathcal{D}} H_{n}\left(k r_{1}\right) \mathrm{e}^{\mathrm{i} n \theta_{1}} \mathrm{e}^{\mathrm{i} \kappa x_{1}} \mathrm{~d} x_{1} \mathrm{~d} y_{1} \quad \text { for } 0<x<a
$$

and $\mathcal{D}$ is the segment of the disc $r_{1}<a$ with $x_{1}<0$.

Now, we let $\kappa \rightarrow k$. Put $\kappa=k+\mathrm{i} \varepsilon$ with $\varepsilon>0$. We have $\mathrm{e}^{\mathrm{i} \kappa x} \simeq(1-\varepsilon x) \mathrm{e}^{\mathrm{i} k x}, k^{2}-\kappa^{2} \simeq-2 \mathrm{i} \varepsilon k$ and $\mathcal{N}_{n}(\kappa a) \simeq 2 \mathrm{i} / \pi+\mathrm{i} \varepsilon k a^{2} d_{n}(k a)$, with $d_{n}$ defined by (59). Hence, in the limit $\varepsilon \rightarrow 0+$, the right-hand side of (124) becomes

$$
\frac{\mathrm{e}^{\mathrm{i} k x}}{\mathrm{i} k^{2}} \sum_{n=-\infty}^{\infty} C_{n}^{(1)}\left\{1-2 \mathrm{i} k x+\pi \mathrm{i}(k a)^{2} d_{n}(k a)\right\} .
$$

Finally, use of (85), (89) and (106) gives the result (122).

Return to (121) and (122), and add them together; the terms in $\mathcal{H}$ cancel, and then (120) gives

$$
\langle u(\mathbf{r})\rangle \mathrm{e}^{\mathrm{i} k x}\left\{1+m_{0} n_{0}(\pi / 4) a^{2}(1-2 \mathrm{i} k x)\right\}, \quad x>a .
$$

This should be compared with $(6),\langle u(\mathbf{r})\rangle=A \mathrm{e}^{\mathrm{i} K x}$. Write

$$
A \simeq 1+m_{0} A_{1}+m_{0}^{2} A_{2} \text { and } \quad K \simeq k+m_{0} k_{1}+m_{0}^{2} k_{2},
$$


so that $K^{2} \simeq k^{2}+2 m_{0} k k_{1}+m_{0}^{2}\left(k_{1}^{2}+2 k k_{2}\right)$. Then,

$$
A \mathrm{e}^{\mathrm{i} K x}=\mathrm{e}^{\mathrm{i} k x}\left\{1+m_{0}\left(A_{1}+\mathrm{i} k_{1} x\right)+m_{0}^{2}\left(A_{2}+\mathrm{i} x\left[A_{1} k_{1}+k_{2}\right]-k_{1}^{2} x^{2} / 2\right)\right\}
$$

the terms involving $m_{0}^{2}$ will be used later. Comparing (127) and (128) gives $A_{1}=\phi / 4$ and $k_{1}=-k \phi / 2$, where $\phi=n_{0} \pi a^{2}$ is the area fraction occupied by the scatterers. Hence, $A \simeq 1+m_{0} \phi / 4$ and $K^{2} \simeq k^{2}\left(1-m_{0} \phi\right)$, in agreement with the Foldy estimates, (84) and (91), correct to first order in $m_{0}$.

The idea of rewriting an expansion such as (127) in the form (6) is well established; see [19, $\S 7.4 .2]$.

\subsection{Second order in $m_{0}$}

At second order in $m_{0}$, we add the last term in (98) to the right-hand side of (119). Using (118), this term becomes

$$
-k^{2} m_{0}^{2} \sum_{i=1}^{N} \sum_{j=1}^{N} \mathrm{e}^{\mathrm{i} k x_{j}} \int_{D_{i}} G_{0}\left(\mathbf{r}, \mathbf{r}^{\prime}\right) I_{1}\left(r_{j}^{\prime}, \theta_{j}^{\prime}\right) \mathrm{d} V^{\prime}=u_{2}(\mathbf{r})
$$

say; recall that $r_{j}, \theta_{j}$ are polar coordinates centred at $\left(x_{j}, y_{j}\right)$, the centre of the $j$ th disc, $D_{j}$.

Evidently, the evaluation of the integrals in (129) will be different if $i=j$ or $i \neq j$, and so we write

$$
u_{2}=u_{2}^{(1)}+u_{2}^{(2)}
$$

where $u_{2}^{(2)}=u_{2}-u_{2}^{(1)}$ and

$$
u_{2}^{(1)}(\mathbf{r})=-k^{2} m_{0}^{2} \sum_{j=1}^{N} \mathrm{e}^{\mathrm{i} k x_{j}} \int_{D_{j}} G_{0}\left(\mathbf{r}, \mathbf{r}^{\prime}\right) I_{1}\left(r_{j}^{\prime}, \theta_{j}^{\prime}\right) \mathrm{d} V^{\prime}
$$

\subsubsection{Calculation of $\left\langle u_{2}^{(1)}\right\rangle$}

From (101), we obtain

$$
u_{2}^{(1)}(\mathbf{r})=m_{0}^{2} \sum_{j=1}^{N} \mathrm{e}^{\mathrm{i} k x_{j}} I_{2}\left(r_{j}, \theta_{j}\right),
$$

where $I_{2}$ is given by (112) for $r_{j}>a$ and by (115) for $r_{j}<a$. Then, proceeding as in Section 7.1, we obtain

$$
\left\langle u_{2}^{(1)}(\mathbf{r})\right\rangle=\mathrm{e}^{\mathrm{i} k x} m_{0}^{2}(\phi / 4)\left\{P_{0}(k a)+(2 \mathrm{i} k x-1) Q_{0}(k a)\right\}, \quad x>a,
$$

where $Q_{0}(k a)=\pi(k a)^{2} \mathcal{H}(k a)$ and

$$
P_{0}(k a)=(k a)^{2}\left[\frac{1}{4}-2 \pi \mathcal{H}(k a)+\frac{\pi \mathrm{i}}{2} \sum_{n=-\infty}^{\infty} \mathcal{J}_{n}(k a) J_{n}(k a) H_{n}(k a)\right]
$$

\subsubsection{Calculation of $\left\langle u_{2}^{(2)}\right\rangle$}

When $i \neq j, r_{j}^{\prime}>a$ (as the scatterers are not allowed to overlap) and so we can use (105) in (102):

$$
\int_{D_{i}} G_{0}\left(\mathbf{r}, \mathbf{r}^{\prime}\right) I_{1}\left(r_{j}^{\prime}, \theta_{j}^{\prime}\right) \mathrm{d} V^{\prime}=-\sum_{n=-\infty}^{\infty} \mathrm{i}^{n} C_{n}^{(1)} \int_{D_{i}} G_{0}\left(\mathbf{r}, \mathbf{r}^{\prime}\right) H_{n}\left(k r_{j}^{\prime}\right) \mathrm{e}^{\mathrm{i} n \theta_{j}^{\prime}} \mathrm{d} V^{\prime}
$$


Here, $r_{j}^{\prime}, \theta_{j}^{\prime}$ are the polar coordinates of the point at $\mathbf{r}^{\prime}$ with respect to the centre of $D_{j}$. So, to integrate over $D_{i}$, we need Graf's addition theorem to express $H_{n}\left(k r_{j}^{\prime}\right) \mathrm{e}^{\mathrm{i} n \theta_{j}^{\prime}}$ in terms of $J_{m}\left(k r_{i}^{\prime}\right) \mathrm{e}^{\mathrm{i} m \theta_{i}^{\prime}}$ :

$$
H_{n}\left(k r_{j}^{\prime}\right) \mathrm{e}^{\mathrm{i} n \theta_{j}^{\prime}}=\sum_{m=-\infty}^{\infty} J_{m}\left(k r_{i}^{\prime}\right) H_{n-m}\left(k R_{j i}\right) \mathrm{e}^{\mathrm{i} m \theta_{i}^{\prime}} \mathrm{e}^{\mathrm{i}(n-m) \alpha_{j i}}
$$

where $x_{i}-x_{j}=R_{j i} \cos \alpha_{j i}$ and $y_{i}-y_{j}=R_{j i} \sin \alpha_{j i}$; see Fig. 1 for a diagram showing notation. Hence,

$$
-k^{2} \int_{D_{i}} G_{0}\left(\mathbf{r}, \mathbf{r}^{\prime}\right) I_{1}\left(r_{j}^{\prime}, \theta_{j}^{\prime}\right) \mathrm{d} V^{\prime}=\sum_{m, n} \mathrm{i}^{n} C_{n}^{(1)} H_{n-m}\left(k R_{j i}\right) \mathrm{e}^{\mathrm{i}(n-m) \alpha_{j i}} L_{m}\left(r_{i}, \theta_{i}\right),
$$

where $L_{n}$ is defined by (103) and we have used the shorthand notation

$$
\sum_{m, n} \equiv \sum_{m=-\infty}^{\infty} \sum_{n=-\infty}^{\infty}
$$

Thus, from (129), (130) and (131), we obtain

$$
u_{2}^{(2)}(\mathbf{r})=m_{0}^{2} \sum_{m, n} C_{n}^{(1)} \Omega_{m n}(\mathbf{r})
$$

with

$$
\Omega_{m n}=\mathrm{i}^{n} \sum_{i=1}^{N} \sum_{\substack{j=1 \\ j \neq i}}^{N} \mathrm{e}^{\mathrm{i} k x_{j}} H_{n-m}\left(k R_{j i}\right) \mathrm{e}^{\mathrm{i}(n-m) \alpha_{j i}} L_{m}\left(r_{i}, \theta_{i}\right) .
$$

To compute the ensemble average of $\Omega_{m n}$, we need a conditional probability. We use $p\left(\mathbf{r}_{i}\right)=n_{0} / N$ and $p\left(\mathbf{r}_{j} \mid \mathbf{r}_{i}\right)=\left(n_{0} / N\right) H\left(R_{i j}-b\right)$, as before; see (17). Then, as the scatterers are indistinguishable, we obtain

$$
\left\langle\Omega_{m n}\right\rangle=\mathrm{i}^{n} n_{0}^{2} \frac{N-1}{N} \int_{B_{N}} L_{m}\left(r_{1}, \theta_{1}\right) \int_{B_{N}} H\left(R_{21}-b\right) \mathrm{e}^{\mathrm{i} k x_{2}} H_{n-m}\left(k R_{21}\right) \mathrm{e}^{\mathrm{i}(n-m) \alpha_{21}} \mathrm{~d} V_{2} \mathrm{~d} V_{1} .
$$

As before, we replace $\mathrm{e}^{\mathrm{i} k x_{2}}$ by $\mathrm{e}^{\mathrm{i} \kappa x_{2}}$ with $\operatorname{Im} \kappa>0$, we let $N \rightarrow \infty$ so that $B_{N}$ becomes the half-plane $x>0$, we evaluate the integrals and then we let $\kappa \rightarrow k$. To begin, we note that the inner integral is very similar to (123); we take its value as

$$
\frac{2}{\mathrm{i} k}(-\mathrm{i})^{n-m} \frac{(\kappa+k) \mathrm{e}^{\mathrm{i} k x_{1}}+\pi \mathrm{i} k \mathrm{e}^{\mathrm{i} \kappa x_{1}} \mathcal{N}_{n-m}(\kappa b)}{k^{2}-\kappa^{2}},
$$

with $\mathcal{N}_{n}$ defined by (125). This result is exact when $x_{1}>b$ but it is an approximation when $0<x_{1}<b$; see the discussion below (124). Using (135) for all $x_{1}>0$, we obtain

$$
\left\langle\Omega_{m n}\right\rangle=\frac{2 n_{0}^{2}}{\mathrm{i} k\left(k^{2}-\kappa^{2}\right)}\left\{(\kappa+k) \mathcal{L}_{m}(k)+\pi \mathrm{i} k \mathcal{N}_{n-m}(\kappa b) \mathcal{L}_{m}(\kappa)\right\}
$$

where

$$
\begin{aligned}
\mathcal{L}_{m}(\kappa) & =\mathrm{i}^{m} \int_{x_{1}>0} \mathrm{e}^{\mathrm{i} \kappa x_{1}} L_{m}\left(r_{1}, \theta_{1}\right) \mathrm{d} x_{1} \mathrm{~d} y_{1} \\
& =\frac{2 C_{m}^{(1)}}{k^{2}-\kappa^{2}}\left\{\frac{\kappa+k}{\mathrm{i} k} \mathrm{e}^{\mathrm{i} k x}+\pi \mathrm{e}^{\mathrm{i} \kappa x} \mathcal{N}_{m}(\kappa a)\right\}+\frac{\pi^{2}}{4} \mathrm{i}^{\mathrm{i} \kappa x} \int_{0}^{a} J_{m}\left(\kappa r_{1}\right) \Lambda_{m}^{(1)}\left(k r_{1}\right) r_{1} \mathrm{~d} r_{1} .
\end{aligned}
$$


Here, we have used (105) and (109), and made calculations similar to (124).

Letting $\kappa \rightarrow k$, we obtain

$$
\lim _{\kappa \rightarrow k}\left\langle\Omega_{m n}\right\rangle=n_{0}^{2} k^{-2} \mathrm{i}\left\{\left[1+\pi \mathrm{i}(k b)^{2} d_{n-m}(k b)\right] \mathcal{L}_{m}(k)-2 k \mathcal{L}_{m}^{\prime}(k)\right\},
$$

where we have used $\mathcal{N}_{m}(k b)=2 \mathrm{i} / \pi$ and $\mathcal{N}_{m}^{\prime}(k b)=k b d_{m}(k b)$. Letting $\kappa \rightarrow k$ in (136) gives

$$
\mathcal{L}_{m}(k)=k^{-2} \mathrm{e}^{\mathrm{i} k x}\left\{p_{m}^{(0)}+(2 \mathrm{i} k x-1) p_{m}^{(1)}\right\},
$$

where $p_{m}^{(0)}=\frac{1}{2} \pi(k a)^{2} J_{m-1}(k a) J_{m+1}(k a)$ and $p_{m}^{(1)}=-\mathrm{i} C_{m}^{(1)}$. Differentiating (136) with respect to $\kappa$ gives

$$
\begin{gathered}
\mathcal{L}_{m}^{\prime}(\kappa) \frac{4 \kappa C_{m}^{(1)}}{\left(k^{2}-\kappa^{2}\right)^{2}}\left\{\frac{\kappa+k}{\mathrm{i} k} \mathrm{e}^{\mathrm{i} k x}+\pi \mathrm{e}^{\mathrm{i} \kappa x} \mathcal{N}_{m}(\kappa a)\right\}+\frac{2 C_{m}^{(1)}}{k^{2}-\kappa^{2}}\left\{\frac{\mathrm{e}^{\mathrm{i} k x}}{\mathrm{i} k}+\pi \mathrm{e}^{\mathrm{i} \kappa x}\left[\mathrm{i} x \mathcal{N}_{m}(\kappa a)+a \mathcal{N}_{m}^{\prime}(\kappa a)\right]\right\} \\
+\frac{\pi^{2}}{4} \mathrm{i} \mathrm{e}^{\mathrm{i} \kappa x} \int_{0}^{a}\left[\mathrm{i} x J_{m}\left(\kappa r_{1}\right)+r_{1} J_{m}^{\prime}\left(\kappa r_{1}\right)\right] \Lambda_{m}^{(1)}\left(k r_{1}\right) r_{1} \mathrm{~d} r_{1} .
\end{gathered}
$$

In the limit $\kappa \rightarrow k$, we find (after some calculation)

$$
2 k \mathcal{L}_{m}^{\prime}(k)=k^{-2} \mathrm{e}^{\mathrm{i} k x}\left\{q_{m}^{(0)}+(2 \mathrm{i} k x-1) q_{m}^{(1)}+(k x)^{2} q_{m}^{(2)}\right\},
$$

where $q_{m}^{(1)}=\mathrm{i} C_{m}^{(1)}+p_{m}^{(0)}, q_{m}^{(2)}=2 \mathrm{i} C_{m}^{(1)}$ and

$$
q_{m}^{(0)}=\pi(k a)^{2} C_{m}^{(1)}\left[d_{m}(k a)-\mathcal{N}_{m}^{\prime \prime}(k a)\right]+p_{m}^{(0)}+\frac{\mathrm{i} \pi^{2}}{2} k^{3} \int_{0}^{a} J_{m}^{\prime}(k r) \Lambda_{m}^{(1)}(k r) r^{2} \mathrm{~d} r .
$$

Simplification gives

$$
\begin{aligned}
q_{m}^{(0)}= & 2 \pi(k a)^{2} C_{m}^{(1)}\left\{d_{m}(k a)-J_{m}(k a) H_{m}(k a)+(\mathrm{i} / \pi)\left[1-m^{2} /(k a)^{2}\right]\right\} \\
& +\left[1+\pi \mathrm{i}(k a)^{2} d_{m}(k a)\right] p_{m}^{(0)}+2 \pi \int_{0}^{k a}\left[J_{m}^{\prime}(x)\right]^{2} x^{3} \mathrm{~d} x .
\end{aligned}
$$

Substituting (138) and (139) in (137) gives

$$
\lim _{\kappa \rightarrow k}\left\langle\Omega_{m n}\right\rangle=\mathrm{i} n_{0}^{2} k^{-4} \mathrm{e}^{\mathrm{i} k x}\left\{P_{m n}^{(0)}+(2 \mathrm{i} k x-1) P_{m n}^{(1)}-(k x)^{2} q_{m}^{(2)}\right\}
$$

where $P_{m n}^{(j)}=\left[1+\pi \mathrm{i}(k b)^{2} d_{n-m}(k b)\right] p_{m}^{(j)}-q_{m}^{(j)}, j=0,1$.

Next, we sum over $m$. From (85) and (106), we have

$$
\sum_{m=-\infty}^{\infty} C_{m}^{(1)}=\frac{\pi \mathrm{i}}{4}(k a)^{2}
$$

Hence, using (87), we obtain

$$
\sum_{m=-\infty}^{\infty} P_{m n}^{(1)}=\frac{\pi}{2}(k a)^{2}+\pi(k b)^{2} \sum_{m=-\infty}^{\infty} C_{m}^{(1)} d_{n-m}(k b)
$$

Then, from (134), we find that

$$
\left\langle u_{2}^{(2)}(\mathbf{r})\right\rangle=\mathrm{e}^{\mathrm{i} k x} m_{0}^{2}\left(\phi^{2} / 4\right)\left\{P_{1}+(2 \mathrm{i} k x-1) Q_{1}+(k x)^{2} R_{1}\right\}
$$


where

$$
\begin{aligned}
P_{1} & =\frac{4 \mathrm{i}}{\pi^{2}(k a)^{4}} \sum_{m, n} C_{n}^{(1)} P_{m n}^{(0)}=\frac{(k b)^{2}}{\mathrm{i}(k a)^{2}} \sum_{m, n} p_{m}^{(0)} \mathcal{J}_{n}(k a) d_{n-m}(k b)+\frac{1}{\pi(k a)^{2}} \sum_{m=-\infty}^{\infty} q_{m}^{(0)}, \\
Q_{1} & =\frac{4 \mathrm{i}}{\pi^{2}(k a)^{4}} \sum_{m, n} C_{n}^{(1)} P_{m n}^{(1)}=-\frac{1}{2}+\frac{4 \mathrm{i}}{\pi} \frac{(k b)^{2}}{(k a)^{4}} \sum_{m, n} C_{m}^{(1)} C_{n}^{(1)} d_{n-m}(k b), \\
R_{1} & =-\frac{4 \mathrm{i}}{\pi^{2}(k a)^{4}} \sum_{m, n} C_{n}^{(1)} q_{m}^{(2)}=-\frac{1}{2} .
\end{aligned}
$$

We add (132) and (142) to the right-hand side of (127), giving the approximation

$$
\begin{aligned}
\langle u(\mathbf{r})\rangle \mathrm{e}^{\mathrm{i} k x}\left\{1+m_{0}(\phi / 4)\left(1+m_{0}\left[P_{0}-Q_{0}\right]+m_{0} \phi\left[P_{1}-Q_{1}\right]\right)\right. & \\
& \left.-\mathrm{i} k x m_{0}(\phi / 2)\left(1-m_{0} Q_{0}-m_{0} \phi Q_{1}\right)-(k x)^{2} m_{0}^{2} \phi^{2} / 8\right\} .
\end{aligned}
$$

When this is compared with (128), we find that $A_{1}=\phi / 4$ and $k_{1}=-k \phi / 2$, as before, the terms in $x^{2}$ agree,

$$
A_{2}=\left(P_{0}-Q_{0}+\phi\left[P_{1}-Q_{1}\right]\right) \phi / 4 \quad \text { and } \quad k_{2} / k=Q_{0} \phi / 2+\left(4 Q_{1}+1\right) \phi^{2} / 8
$$

Hence, we obtain the approximation

$$
K^{2} / k^{2} \simeq 1-m_{0} \phi+m_{0}^{2} \phi Q_{0}+m_{0}^{2} \phi^{2}\left(Q_{1}+1 / 2\right) .
$$

The last term implies that $\delta_{2}$ in (3) is given by

$$
\delta_{2} \simeq k^{2} m_{0}^{2}\left(\pi a^{2}\right)^{2}\left(Q_{1}+1 / 2\right)=4 \pi \mathrm{i} m_{0}^{2} b^{2} \sum_{m, n} C_{m}^{(1)} C_{n}^{(1)} d_{n-m}(k b)
$$

where we have used (144). Then, using (106), we find precise agreement with (93). We also find the approximation

$$
A \simeq 1+m_{0} \phi / 4+m_{0}^{2}\left(P_{0}-Q_{0}\right) \phi / 4+m_{0}^{2}\left(P_{1}-Q_{1}\right) \phi^{2} / 4
$$

The term involving $m_{0}^{2} \phi$ in (146) differs from the Foldy approximation, (91), by the presence of $P_{0}$. This quantity came from an exact calculation of $\left\langle u_{2}^{(1)}\right\rangle$; see (132). Specifically, $P_{0}$ came from a certain integral over the interior of a typical scatterer. We have checked that $P_{0}$ does not vanish identically [17].

\subsection{Comparison with "point scatterers"}

Similar results have been obtained by Maurel [18] for uncorrelated configurations of "point scatterers". For the deterministic problem, the governing partial differential equation is

$$
\left(\nabla^{2}+k^{2}\right) u=k^{2} m_{0} \pi a^{2} u(\mathbf{r}) \sum_{i=1}^{N} \delta\left(\mathbf{r}-\mathbf{r}_{i}\right),
$$

where $\delta$ is the Dirac delta function. The factor $\pi a^{2}$ ensures agreement with the right-hand side of (97): $\int u \chi_{i} \mathrm{~d} V \simeq \pi a^{2} u\left(\mathbf{r}_{i}\right)$ when $D_{i}$ is small. Maurel [18] found the approximation

$$
\langle u(\mathbf{r})\rangle=\mathrm{e}^{\mathrm{i} k x}\left\{1+m_{0}(\phi / 4)\left[1-m_{0} \mathcal{Q}+m_{0} \phi / 2\right]-\mathrm{i} k x m_{0}(\phi / 2)\left[1-m_{0} \mathcal{Q}+m_{0} \phi / 2\right]-(k x)^{2} m_{0}^{2} \phi^{2} / 8\right\},
$$


where $\mathcal{Q}(k a)=\pi(\mathrm{i} / 4)(k a)^{2} H_{0}(k a)$. From (147), the estimates

$$
\begin{aligned}
A & \simeq 1+m_{0} \phi / 4-m_{0}^{2}(\phi / 4) \mathcal{Q}+m_{0}^{2} \phi^{2} / 8, \\
K / k & \simeq 1-m_{0} \phi / 2+m_{0}^{2}(\phi / 2) \mathcal{Q}-m_{0}^{2} \phi^{2} / 8
\end{aligned}
$$

follow; squaring the last formula gives $K^{2} / k^{2} \simeq 1-m_{0} \phi+m_{0}^{2} \phi \mathcal{Q}$, with no term in $m_{0}^{2} \phi^{2}$.

Intuitively, the point-scatterer limit should correspond to $k a \rightarrow 0$. If we compare (145) and (147), we see that we will have agreement if

$$
P_{0}-Q_{0} \sim-\mathcal{Q}, \quad P_{1}-Q_{1} \sim \frac{1}{2}, \quad Q_{0} \sim \mathcal{Q} \text { and } Q_{1} \sim-\frac{1}{2} .
$$

The third of these follows quickly from the definition of $Q_{0}$, whereas the first follows from the fact that $P_{0}$ is smaller than $Q_{0}$ as $k a \rightarrow 0$. The second and fourth of (148) involve $P_{1}$ and $Q_{1}$, and these depend on $k b$ as well as $k a$. From (144), we find that

$$
Q_{1}+1 / 2 \sim-\pi(\mathrm{i} / 4)(k b)^{2} d_{0}(k b) \quad \text { as } k a \rightarrow 0 ;
$$

the limiting value vanishes when $k b \rightarrow 0$, which is the appropriate limit for uncorrelated scatterers. Finally, consider $P_{1}$, defined by (143) as a double-sum term plus a single-sum term. In the double sum, the dominant contributions come from $\mathcal{J}_{0} \sim 1, p_{0}^{(0)} \sim(\pi / 8)(k a)^{4}$ and $p_{ \pm 1}^{(0)} \sim p_{0}^{(0)} / 2$ as $k a \rightarrow 0$. Hence, asymptotically, the double-sum term is

Making use of (140), (141),

$$
-\mathrm{i}(\pi / 8)(k a)^{2}(k b)^{2}\left[d_{0}(k b)+d_{1}(k b)\right] \rightarrow 0 \quad \text { as } k a \rightarrow 0 .
$$

$$
\sum_{m=-\infty}^{\infty} p_{m}^{(0)}=0 \text { and } \sum_{m=-\infty}^{\infty}\left[J_{m}^{\prime}(x)\right]^{2}=\frac{1}{2}
$$

the single-sum term in (143),

$$
\frac{1}{\pi(k a)^{2}} \sum_{m=-\infty}^{\infty} q_{m}^{(0)}=-\frac{1}{4}(k a)^{2}+\sum_{m=-\infty}^{\infty}\left\{\mathcal{J}_{m}(k a) \gamma_{m}(k a)+\mathrm{i} d_{m}(k a) p_{m}^{(0)}\right\}
$$

exactly, where $\gamma_{m}(x)=(\mathrm{i} \pi / 2) x^{2}\left[d_{m}(x)-J_{m}(x) H_{m}(x)\right]+m^{2} / 2$. We have $\gamma_{0}(x) \sim x^{2} / 2, d_{0}(x) \sim H_{0}(x)$, $\gamma_{m}(x) \sim|m|+m^{2} / 2$ and $x^{2} d_{m}(x) \sim 2 \mathrm{i}|m| / \pi$ for $m \neq 0$, so that the largest terms in (149) are $O\left((k a)^{2}\right)$ as $k a \rightarrow 0$. Thus, all of (148) hold in the limit $k a \rightarrow 0$, provided $k b \rightarrow 0$ too. It follows that we recover the point-scatterer results if we allow the scatterers to shrink $(k a \rightarrow 0)$ and to become uncorrelated.

\section{Summary}

We conclude with a summary of the various theories. We distinguish between theories of Foldy-Lax type and iterative theories.

\subsection{Foldy-Lax theories}

The simplest is "basic" Foldy theory: it predicts that $K^{2}=k^{2}-4 \mathrm{i} n_{0} f(0)$ and that $A$ is given by (7); it is first order in $n_{0}$; it assumes that the scatterers are statistically independent (possible overlaps are ignored at first order); it uses the forward-scattered far-field pattern for one scatterer, $f(0)$, calculated (exactly) taking proper account of the interior wavefield and density differences, if present; and it makes essential use of the Foldy closure assumption.

As a special case, we have "weak Foldy". This occurs when the scattering is weak, meaning that each scatterer is penetrable with a sound-speed that is close to that in the exterior; this closeness is measured by the 
small parameter, $m_{0}$. (For simplicity, we do not permit density differences here.) The "weak-Foldy" results are given in Section 4.2; in particular, when $u_{\text {in }}=\mathrm{e}^{\mathrm{i} k x}$,

$$
|A|=1+m_{0} \phi / 4-m_{0}^{2}(\phi / 4) \operatorname{Re} Q_{0}
$$

and $K_{\mathrm{i}} / k=m_{0}^{2}(\phi / 2) \operatorname{Im} Q_{0}$, where $K_{\mathrm{i}}=\operatorname{Im} K, \phi=n_{0} \pi a^{2}$ is the filling fraction or area fraction occupied by the scatterers, $Q_{0}(k a)=\pi(k a)^{2} \mathcal{H}$ and $\mathcal{H}(k a)$ is given by (89). Note that

$$
\frac{K_{\mathrm{i}}}{k}=m_{0}^{2} \phi(k a)^{2} \frac{\pi}{8} \sum_{n=-\infty}^{\infty} \mathcal{J}_{n}^{2}(k a)
$$

which is positive, implying attenuation with $x$.

Going beyond Foldy, we can seek corrections proportional to $n_{0}^{2}$. This is more difficult because paircorrelations must be used in order to prevent finite-sized scatterers from overlapping during averaging. The "Linton-Martin" correction to basic Foldy for $K$ is $n_{0}^{2} \delta_{2}$ with $\delta_{2}$ given by (65). It was derived using the Lax QCA as closure assumption. Again, as with basic Foldy, there is a weak version; it gives a term in $n_{0}^{2} m_{0}^{2}$. See Section 4.3. The Linton-Martin theory does not give any estimate for $A$.

Note that Foldy and Linton-Martin theories do not assume explicitly that $k a$ is small. However, letting $k a \rightarrow 0$ gives results for very small scatterers, and these can be compared with results for so-called "point scatterers"; this was done in Section 7.3. In particular, the point-scatterer limit gives

$$
|A|=1+m_{0} \phi / 4-m_{0}^{2}(\phi / 4) \operatorname{Re} \mathcal{Q}+m_{0}^{2} \phi^{2} / 8
$$

and $K_{\mathrm{i}} / k=m_{0}^{2}(\phi / 2) \operatorname{Im} \mathcal{Q}$, where $\mathcal{Q}=\pi(\mathrm{i} / 4)(k a)^{2} H_{0}(k a)$ so that

$$
\begin{aligned}
& \operatorname{Re} \mathcal{Q}=-(\pi / 4)(k a)^{2} Y_{0}(k a) \sim-(1 / 2)(k a)^{2} \log k a, \\
& \operatorname{Im} \mathcal{Q}=(\pi / 4)(k a)^{2} J_{0}(k a) \sim(\pi / 4)(k a)^{2} .
\end{aligned}
$$

\subsection{Iterative theories}

In this paper, we began with the Lippmann-Schwinger equation, which we solved by iteration for weak scattering. At both first and second order in $m_{0}$, we obtained exactly the same expressions for $K$ as those obtained by "weak Foldy" and "weak Linton-Martin". No closure assumptions were used. However, there is one difference in the result for $A$ :

$$
|A|=1+m_{0} \phi / 4-m_{0}^{2}(\phi / 4) \operatorname{Re}\left(Q_{0}-P_{0}\right)+m_{0}^{2}\left(\phi^{2} / 4\right) \operatorname{Re}\left(P_{1}-Q_{1}\right) .
$$

Thus, the term $P_{0}$ is absent from (150). Also,

$$
\frac{K_{\mathrm{i}}}{k}=m_{0}^{2} \phi(k a)^{2} \frac{\pi}{8}\left\{\sum_{n=-\infty}^{\infty} \mathcal{J}_{n}^{2}(k a)-\phi(b / a)^{2} \sum_{m, n} \mathcal{J}_{m}(k a) \mathcal{J}_{n}(k a) \mathcal{J}_{n-m}(k b)\right\} ;
$$

this expression can become negative, so that it fails for values of $\phi$ that are sufficiently large. Equations (155) and (156) do reduce to the point-scatterer limits when both $k a$ and $k b \rightarrow 0$.

\section{REFERENCES}

[1] C. Aristégui and Y. C. Angel, New results for isotropic point scatterers: Foldy revisited, Wave Motion 36 (2002) $383-399$.

[2] S. K. Bose and A. K. Mal, Longitudinal shear waves in a fiber-reinforced composite, Int. J. Solids Struct. 9 (1973) $1075-1085$.

[3] O. P. Bruno and E. M. Hyde, An efficient, preconditioned, high-order solver for scattering by two-dimensional inhomgeneous media, J. Comp. Phys. 200 (2004) 670-694. 
[4] A. Derode, V. Mamou and A. Tourin, Influence of correlations between scatterers on the attenuation of the coherent wave in a random medium, Phys. Rev. E 74 (2006) 036606.

[5] J. G. Fikioris and P. C. Waterman, Multiple scattering of waves. II. "Hole corrections" in the scalar case, J. Math. Phys. 5 (1964) 1413-1420.

[6] L. L. Foldy, The multiple scattering of waves. I. General theory of isotropic scattering by randomly distributed scatterers, Phys. Rev. 67 (1945) 107-119.

[7] I. S. Gradshteyn and I. M. Ryzhik, Tables of Integrals, Series and Products, 6th edn, Academic Press, New York, 2000.

[8] F. C. Karal, Jr. and J. B. Keller, Elastic, electromagnetic, and other waves in a random medium, J. Math. Phys. 5 (1964) $537-547$.

[9] M. Lax, Multiple scattering of waves, Rev. Modern Phys. 23 (1951) 287-310.

[10] M. Lax, Multiple scattering of waves. II. The effective field in dense systems, Phys. Rev. 85 (1952) 621-629.

[11] C. M. Linton and P. A. Martin, Multiple scattering by random configurations of circular cylinders: second-order corrections for the effective wavenumber, J. Acoust. Soc. Amer. 117 (2005) 3413-3423.

[12] C. M. Linton and P. A. Martin, Multiple scattering by multiple spheres: a new proof of the Lloyd-Berry formula, SIAM J. Appl. Math. 66 (2006) 1649-1668.

[13] C. M. Linton and P. McIver, Handbook of Mathematical Techniques for Wave/Structure Interactions, Chapman \& Hall/CRC, Boca Raton, 2001.

[14] P. Lloyd and M. V. Berry, Wave propagation through an assembly of spheres IV. Relations between different multiple scattering theories, Proc. Phys. Soc. 91 (1967) 678-688.

[15] P. A. Martin, Acoustic scattering by inhomogeneous obstacles, SIAM J. Appl. Math. 64 (2003) $297-308$.

[16] P. A. Martin, Multiple Scattering, Cambridge University Press, 2006.

[17] P. A. Martin and A. Maurel, Multiple scattering by random configurations of circular cylinders: weak scattering without closure assumptions, Wave Motion 45 (2008) 865-880.

[18] A. Maurel, Reflection and transmission by a slab with randomly distributed isotropic point scatterers. Preprint, 2008.

[19] A. H. Nayfeh, Perturbation Methods, Wiley, New York, 2000.

[20] P. R. Siqueira and K. Sarabandi, Method of moments evaluation of the two-dimensional quasi-crystalline approximation, IEEE Trans. Antennas 83 Propag. 44 (1996) 1067-1077.

[21] L. Tsang, J. A. Kong, K. -H. Ding and C. O. Ao, Scattering of Electromagnetic Waves: Numerical Simulations, Wiley, New York, 2001.

[22] V. Twersky, On scattering of waves by random distributions. I. Free-space scatterer formalism, J. Math. Phys. 3 (1962) $700-715$.

[23] V. Twersky, Acoustic bulk parameters in distributions of pair-correlated scatterers, J. Acoust. Soc. Am. 64 (1978) $1710-1719$.

[24] P. C. Waterman and R. Truell, Multiple scattering of waves, J. Math. Phys. 2 (1961) 512-537.

[25] R. L. Weaver, A variational principle for waves in discrete random media, Wave Motion 7 (1985) 105-121. 